\title{
Suprathermal electron driven fishbone instability in the TCV tokamak
}

\author{
D. Choi ${ }^{1}$, A. Merle ${ }^{1}$, S. Coda ${ }^{1}$, J. Decker ${ }^{1}$, J. P. Graves ${ }^{1}$, \\ A. S. Tema Biwole ${ }^{1}$, L. Porte $^{1}$, Y. Peysson ${ }^{2}$, and and the TCV team*1 \\ ${ }^{1}$ Ecole Polytechnique Fédérale de Lausanne (EPFL), Swiss Plasma Center (SPC), \\ CH-1015 Lausanne, Switzerland \\ ${ }^{2}$ CEA, IRFM, F-13108, Saint-Paul-lez-Durance, France
}

\begin{abstract}
Observations of fishbone oscillations driven by energetic electrons were reported in several tokamaks, but experimental evidence of the interaction between electrons and the mode has remained limited. In the TCV tokamak, a state-of-the-art multi-channel hard X-ray spectroscopy system equipped with digital pulse detection permits studies of redistribution of ECCD-created suprathermal electrons at high temporal resolution. For the first time, the response of hard X-ray profiles to the internal kink mode is observed, at the frequency of the mode. This work demonstrates the role of suprathermal electrons in destabilizing the fishbone mode and in particular the interaction of trapped electrons with the mode. The mode is destabilized after the ramp-up of suprathermal electrons. Then trapped suprathermal electrons preferentially interact with the mode and are expelled during the mode oscillation phase. The experimentally observed mode frequency and rotation direction are in good agreement with the solution of the linear fishbone dispersion relation, where the electron distribution function is modelled using a Fokker-Planck code coupled with a hard X-ray synthetic diagnostic. The frequency and stability predicted by the modeling also agree well with data from an ECCD power scan, as the varying suprathermal electron distribution affects the mode behavior.
\end{abstract}

\section{Introduction}

Suprathermal electrons generated by means of radio-frequency $(\mathrm{RF})$ heating and current drive can trigger plasma instabilities in magnetically confined fusion experiments [1-6], which in turn redistributes particles and may deteriorate plasma performance. Electron fishbones, an energetic electron driven instability, have been observed during electron cyclotron resonance heating or current drive (ECRH or ECCD) [1,6-10], during lower hybrid current drive (LHCD) [11-14], and when both ECRH and LHCD are used [7]. The excitation of this internal kink was attributed to the resonant interaction between fast electrons and the mode. In LHCD discharges at high collisionality, observation of fishbone-like oscillations was explained by non-resonant destabilization due to the suprathermal electron pressure $[15,16]$.

*See author list of S. Coda et al 2019 Nucl. Fusion 59112023 
In the experimental investigation of electron fishbones, diagnosing the suprathermal electron population is a primary goal to assess the stability of the mode and possible loss of energetic electrons. However, suprathermal electron dynamics related to the fishbone oscillation is still only partially understood. While hard X-ray diagnostics have been the main tool to study the temporal evolution of suprathermal electron energy spectra and spatial structure $[8,9,12,13,17]$, there has been no detailed analysis at a frequency comparable with the mode frequency, rather only in a few separate time windows during a single fishbone burst [12]. Electron cyclotron emission (ECE) radiated by suprathermal electrons has also showed a correlation between the mode and the fast electron population with higher time resolution, but the measurement lacks spatial resolution [15].

In this work, we report new experimental data on the fishbone instability, driven by suprathermal electrons during ECCD discharges in the TCV tokamak. A newly developed ECCD scenario enables collecting hard X-ray photons over regular electron fishbone bursts. Thus the temporal resolution of the hard X-ray measurement is enhanced to resolve the observed mode frequency. The kink mode structure is observed in the hard X-ray profiles, as well as in the soft X-ray profiles and the electron temperature profiles measured by the Thomson scattering system. In addition, the hard X-ray camera that can rotate on its axis permits a study of the different responses of trapped and passing electrons to the $\mathrm{m} / \mathrm{n}=1 / 1$ mode. The stability of the mode is assessed by solving the linear fishbone dispersion relation, using a realistic electron distribution function modelled by solving the Fokker-Planck equation, with the aid of a hard X-ray synthetic diagnostic.

This paper is arranged as follows: in section 2 we describe the experimental setup and diagnostics in the TCV tokamak. The experimental observation of the electron fishbones and detailed analysis are presented in section 3. In section 4, electron fishbone characteristics with varying ECCD power and location are analyzed. The experimental observations are compared to the solution of the linear fishbone dispersion equation, coupled with the Fokker-Planck simulation, in section 5 . Conclusions can be found in section 6 .

\section{Experimental setup}

TCV [18] is a medium-sized tokamak $(\mathrm{R}=0.88 \mathrm{~m}, \mathrm{a}=0.25 \mathrm{~m})$ that features a high-power density ECRH/ECCD system. In the discharges analyzed in this article, one $82.7 \mathrm{GHz}$ gyrotron was applied with a power of up to $750 \mathrm{~kW}$, heating at the second (X2) harmonic of the electron cyclotron resonance. The typical plasma parameters used in this work are: plasma current $|I p| \sim 0.2 \mathrm{MA}$, toroidal magnetic field $\left|B_{\phi}\right| \sim 1.34 \mathrm{~T}$, electron density $n_{e} \sim 2.2 \times 10^{19} / \mathrm{m}^{3}$, electron temperature $T_{e} \sim 2.4 \mathrm{keV}$, ion temperature $T_{i} \sim 0.45 \mathrm{keV}$, plasma toroidal rotation frequency $\left|f_{\phi}\right| \sim 4.5 \mathrm{kHz}$ and effective charge $Z_{\text {eff }} \sim 1.2$. The common direction of $I_{p}, B_{\phi}$, and $f_{\phi}\left(v_{\phi}\right)$ is indicated in Figure 1 (a): in the clockwise direction on the top view of TCV. No neutral beam injection system was used. The plasma equilibrium is reconstructed from magnetic measurements, by solving the Grad-Shafranov equation with the code LIUQE [19].

A toroidal magnetic probe array and a soft X-ray tomographic system (XTOMO) are used to analyze toroidal and poloidal mode structures, respectively. The toroidal magnetic probe array [20] comprises three $(\mathrm{z}=-0.23 \mathrm{~m}, \mathrm{z}=0 \mathrm{~m}, \mathrm{z}=0.23 \mathrm{~m})$ high-field-side arrays of 8 probes each at the inner wall and three $(\mathrm{z}=-0.23 \mathrm{~m}, \mathrm{z}=0 \mathrm{~m}, \mathrm{z}=0.23 \mathrm{~m})$ low-field-side arrays of 16 probes each at the outer wall. The magnetic pick-up coils acquire data at $250 \mathrm{kHz}$. The soft 

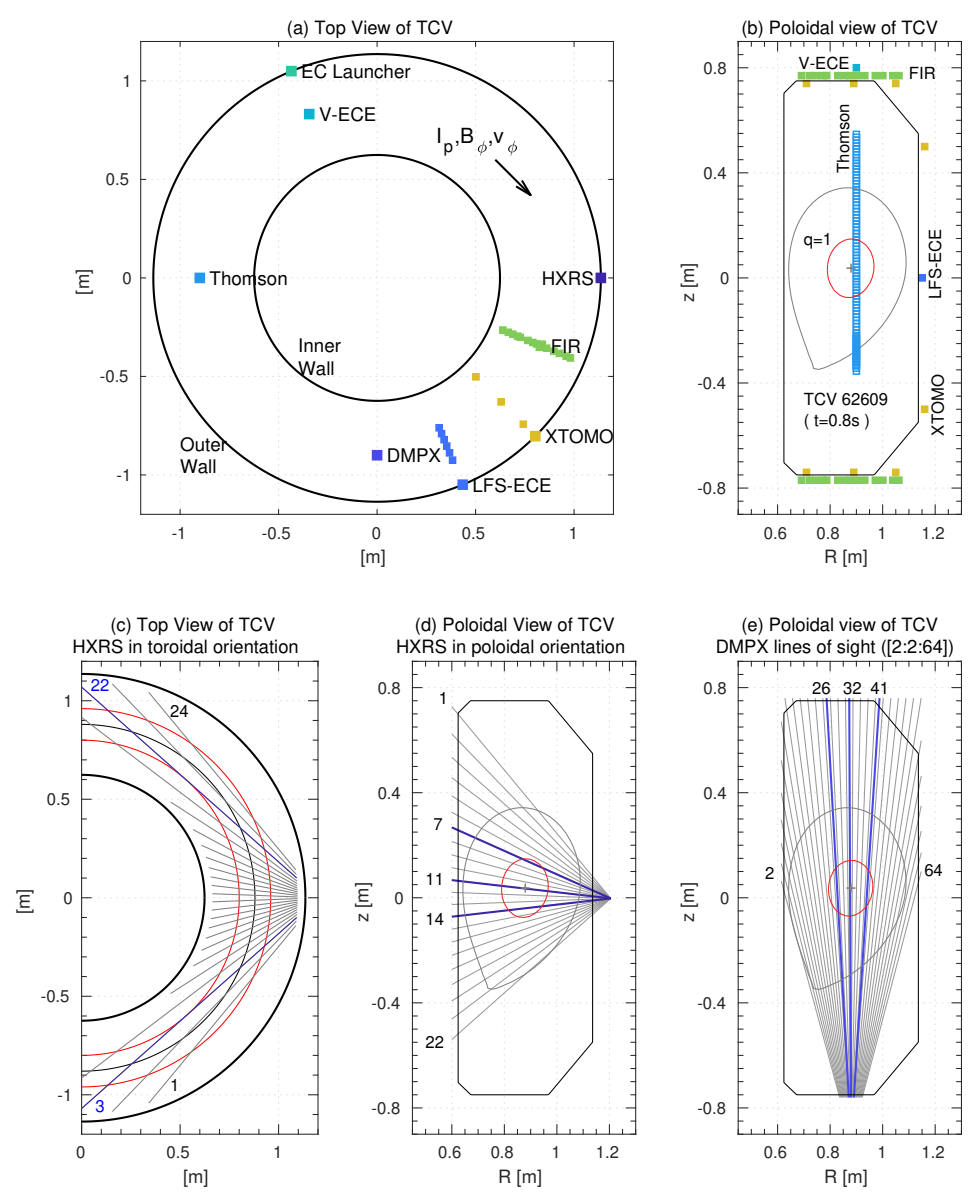

Figure 1: (a) Top view of the diagnostic set in TCV. (b) Poloidal view of the diagnostics. The last closed flux surface and the $\mathrm{q}=1$ surface are presented from TCV shot 62609 . (c,d) Lines of sight of HXRS equatorial camera in the toroidal and poloidal orientations. (e) Lines of sight of DMPX.

X-ray tomographic system (XTOMO) consists of 8 pinhole cameras with 20 p-n junction silicon photodiodes each, acquiring data at $100 \mathrm{kHz}$. The measured signal is used for the tomographic inversion of soft X-ray emission using a finite element grid discretization on the flux surfaces and the minimum Fisher regularization inversion method [21]. The data from both diagnostics are analyzed using the singular value decomposition (SVD) technique to study the time evolution (chronos) of the spatial mode structure (topos) [21,22]. The mode frequency measured in the laboratory frame is converted to the frequency in the plasma frame using plasma toroidal rotation data acquired from Charge Exchange Recombination Spectroscopy (CXRS), which uses the $C_{6+}$ impurity line assumming $v_{\phi}=v_{C 6+}$. The CXRS also provides $T_{i}$ and $Z_{\text {eff }}$.

The fishbone fluctuation of thermal electrons is analyzed in detail using the multi-channel soft X-ray detector (DMPX) and the low field side ECE diagnostic (LFS-ECE). DMPX [23] has a high time resolution of $5 \mu \mathrm{s}$ and a high spatial resolution defined by $7.9 \mathrm{~mm}$ mean distance between two lines of sight, which allows observing changes in the soft X-ray profile in the plasma core far inside the $\mathrm{q}=1$ surface. One-half of the lines of sight (every other chord) are plotted in Figure 1(e), out of a total of 64 channels. Observations of the small scale mode structure inside the $\mathrm{q}=1$ surface are complemented by a recently upgraded Thomson scattering system, 
which provides a high spatial resolution $(6-12 \mathrm{~mm})$ vertical profile of $n_{e}$ and $T_{e}$ at $\mathrm{R}=0.9$ $\mathrm{m}$. The measurement points are plotted in 1(b). For the analysis of the electron temperature fluctuations, the second harmonic X-mode LFS-ECE diagnostic is used, with 24 channels from 65 to $99 \mathrm{GHz}$ [24]. The data is acquired at $200 \mathrm{kHz}$. Another ECE system, vertical ECE, that is positioned at the top of the tokamak (Figure $1(\mathrm{a}, \mathrm{b}), \mathrm{V}-\mathrm{ECE})$ can receive electron cyclotron radiation emitted by suprathermal electrons at the third harmonic of the electron cyclotron frequency, with 12 channels from $89 \mathrm{GHz}$ to $114 \mathrm{GHz}$.

A state-of-the-art hard X-ray tomographic spectrometer (HXRS) [25] is able to detect the Bremsstrahlung radiation of energetic electrons, from $\sim 15 \mathrm{keV}$ to $\sim 200 \mathrm{keV}$. The system comprises four cameras with $24 \mathrm{CdTe}$ detectors each, therefore 96 lines of sights can cover the poloidal plane for the tomographic inversion of the hard X-ray emission distribution. In this work, a single camera located at the midplane $(\mathrm{z}=0 \mathrm{~m})$ has been used. The camera can be rotated by 90 degrees, thus the lines of sight are able to cover either the toroidal plane, parallel to the magnetic field lines (Figure 1 (c)), or the poloidal plane, perpendicular to the magnetic field lines (Figure 1 (d)). Since energetic electrons emit hard X-ray radiation preferentially in the direction of travel (relativistic headlight effect [26]), trapped and passing electrons contribute differently to the hard X-ray photons received by the camera in different orientations. This fact is used in section 3.4, where the response of trapped electrons is studied separately from that of passing electrons.

The TCV hard X-ray system features a digital pulse processing [27], which enables up to 600,000 photon counts per second. After the pulse processing, the photon counts are sorted into user-set energy and time bins. In this work, photon counts are conditionally averaged over repetitive fishbone bursts, in order to enhance the photon statistics in short time bins. This has enabled a mode oscillation of a few $\mathrm{kHz}$ frequency to be directly observed in hard X-ray profiles for the first time.

Each photon impinging on a HXRS detector generates a charge pulse through photon-matter interaction, which is recorded by the associated electronics; the height of the pulse can then be translated into an energy value, based on the calibration performed using radioactive sources. While the photo-electric effect has the largest influence on the detected signal, the low energy contribution from the Compton effect needs to be considered as well. Because of this and, even more importantly, the continuous energy spectrum of the Bremsstrahlung radiation, the electron distribution function cannot be reconstructed directly from the hard X-ray measurement. This limitation can be compensated by numerical modeling: in particular, Fokker-Planck modeling and a hard X-ray synthetic diagnostic have been used for this purpose. The modelled distribution function can also be used to solve the linear fishbone dispersion relation to assess the stability of the mode. The numerical modeling approach is described in detail in section 5 .

\section{Experimental observation of the fishbone oscillation}

In this section we present the experimental observations and detailed analysis of the electron fishbone oscillation. An overview of the experimental conditions and observations is presented in section 3.1. Conditional averaging results of the hard X-ray and other diagnostics over regular fishbone bursts are presented in section 3.2, and the Abel-inversion of the hard X-ray and soft X-ray profiles is discussed in section 3.3. The different behavior of passing and trapped electrons is investigated from the hard X-ray data in section 3.4. 


\subsection{The $\mathrm{m} / \mathrm{n}=1 / 1$ mode excitation during ECCD}

Regular frequency chirping bursts such as those shown in Figure 2 have appeared in the magnetic probe, soft X-ray and ECE data during the ECCD phase in the discharge \#62609, from $0.5 \mathrm{~s}$ to $1.9 \mathrm{~s}$. In this discharge, $530 \mathrm{~kW}$ of co-ECCD power was injected obliquely with a toroidal angle of $20^{\circ}\left(0^{\circ}\right.$ corresponds to pure ECH) with absorption localized at $\rho_{\psi} \sim 0.25$ ( $\rho_{\psi}$ is the square root of the normalized poloidal flux) on the high field side, according to the C3PO ray tracing calculations [28]. The $\mathrm{q}=1$ surface is located at $\rho_{\psi} \sim 0.44$ [19].

Figure 2 (b) shows the Fourier spectrogram of the $n=1$ mode amplitude ( $\mathrm{n}$ is the toroidal mode number), which corresponds to the dominant component (Chronos 1, Figure 2 (a)) from the SVD analysis of the toroidal magnetic probe array signal. The mode rotates in the precessional drift direction of deeply trapped electrons, which is opposite to the plasma current direction. The SVD analysis of the tomographically inverted soft X-ray emission from XTOMO has shown that the mode has a poloidal $\mathrm{m}=1$ structure ( $\mathrm{m}$ is the poloidal mode number), rotating near the $\mathrm{q}=1$ surface in the electron diamagnetic drift direction.

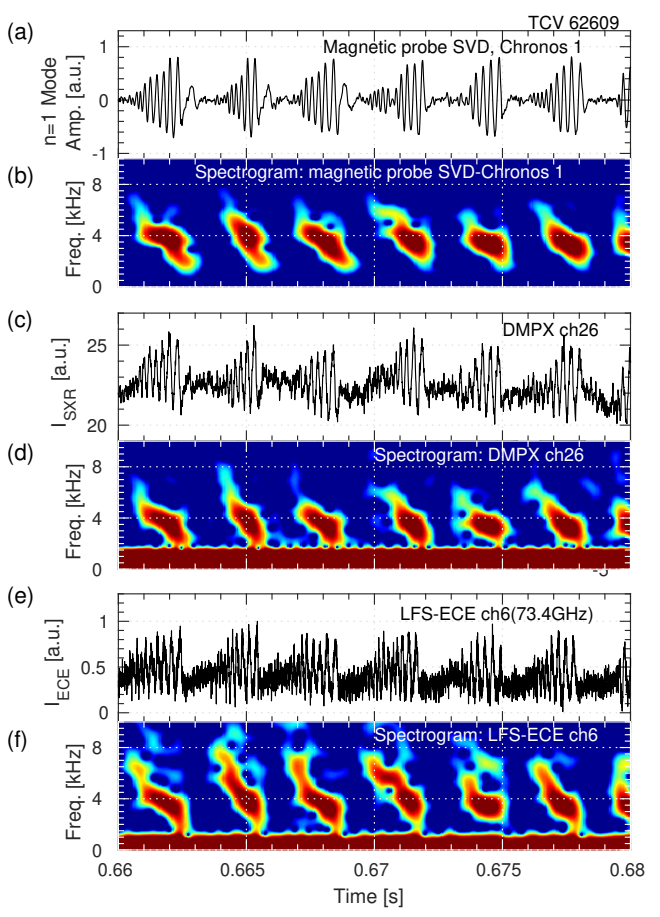

Figure 2: Fishbone shaped oscillations and the Fourier spectrogram of $(a, b)$ magnetic field fluctuation, (c,d) soft X-ray signal, and (e,f) ECE signal.

The spectrograms in Figure 2 show that the mode frequency chirps down from $\sim 5 \mathrm{kHz}$ to $\sim 2$ $\mathrm{kHz}$, during $\sim 2 \mathrm{~ms}$, every $\sim 3 \mathrm{~ms}$. The toroidal rotation frequency in this discharge is $4.5 \pm 0.6$ $\mathrm{kHz}$ in the plasma current direction, which is opposite to the mode rotation direction. Therefore the mode frequency in the plasma frame chirps down from $\sim 9.5 \mathrm{kHz}$ to $\sim 6.5 \mathrm{kHz}$. The regular burst time points are recorded for the conditional averaging of the results.

The time history of soft X-ray profiles in Figure 3 shows that the fishbone-shaped perturbation appear in the off-axis channels, whose lines of sight are parallel to the flux surfaces near the $\mathrm{q}$ $=1$ surface. The lines of sight of DMPX are presented in Figure 1 (e) with the $q=1$ surface of this discharge. The oscillations are accompanied by a central plasma relaxation, as seen in 
(a)

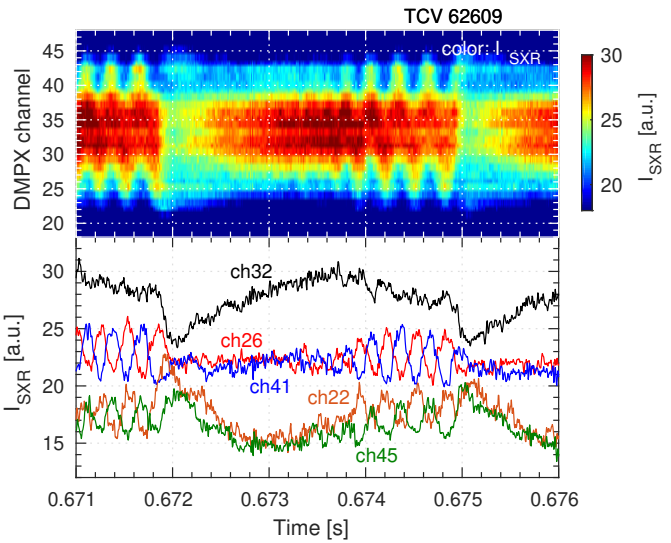

Figure 3: Time history of (a) soft X-ray profiles and (b) the signal of selected channels.

the central soft X-ray signal, $I_{S X R}$ of ch32, taking the form of so-called non-standard sawtooth, classified as saturated or inverted [29].

The first investigations into the non-standard sawtooth [29] found that the sawtooth shape changed during EC heating, depending on EC heating conditions and plasma parameters, such as plasma shape or safety factor. The non-standard sawtooth activity was further studied about 20 years ago to simulate the electron temperature evolution using a magnetic island model in the presence of localized heat sources [30]; however, the spatial mode structure during the plasma relaxation was supported by limited experimental evidence. The mechanism behind the onset of the phenomena remained uncertain. In the present work, experimental evidence for the $\mathrm{m} / \mathrm{n}=1 / 1$ mode structure is provided in section 3.2, and a link between the central plasma relaxation and the mode oscillation is investigated in section 3.3.

At the end of the oscillation phase in Figure 3, there is a collapse of the central soft X-ray profile, resembling a standard sawtooth crash. Since the fishbone-like oscillation exists before the crash, no distinctive sawtooth precursor is observed. Then one must question whether this fishbone-like oscillation is actually an extended sawtooth precursor or whether this instability induces the sawtooth crash at the end of its oscillation. The possibility of the extended sawtooth precursor can be excluded because this fishbone-like oscillation is sometimes observed separately from a sawtooth precursor depending on the ECCD location, as can be seen in section 4.2, Figure 15. Thus it is possible that a small-amplitude sawtooth precursor overlaps with the fishbone-like oscillation. In section 5, we solve the linear fishbone dispersion relation, to study the stability of the fishbone mode of this discharge.

\subsection{Conditional averaging of data}

In order to observe the high frequency suprathermal electron dynamics, the hard X-ray photon counts have been collected over regular fishbone-like bursts. Although the fishbone-like bursts appear regularly, the amplitudes and the lengths of the oscillations vary; thus bursts that have similar amplitude and length have been selected in order to reduce the statistical error.

Conditionally averaged magnetic field fluctuations, ECE signal $I_{E C E}$, soft X-ray intensity $I_{S X R}$ and hard X-ray count rate $I_{H X R}$ are presented in Figure 4 . In the discharge \#62609, the time points of 117 bursts are collected. The relative time variable $\tau$ is introduced such that at $\tau=0$ the mode amplitude from the magnetic probe analysis has a maximum value. $I_{S X R}$ 

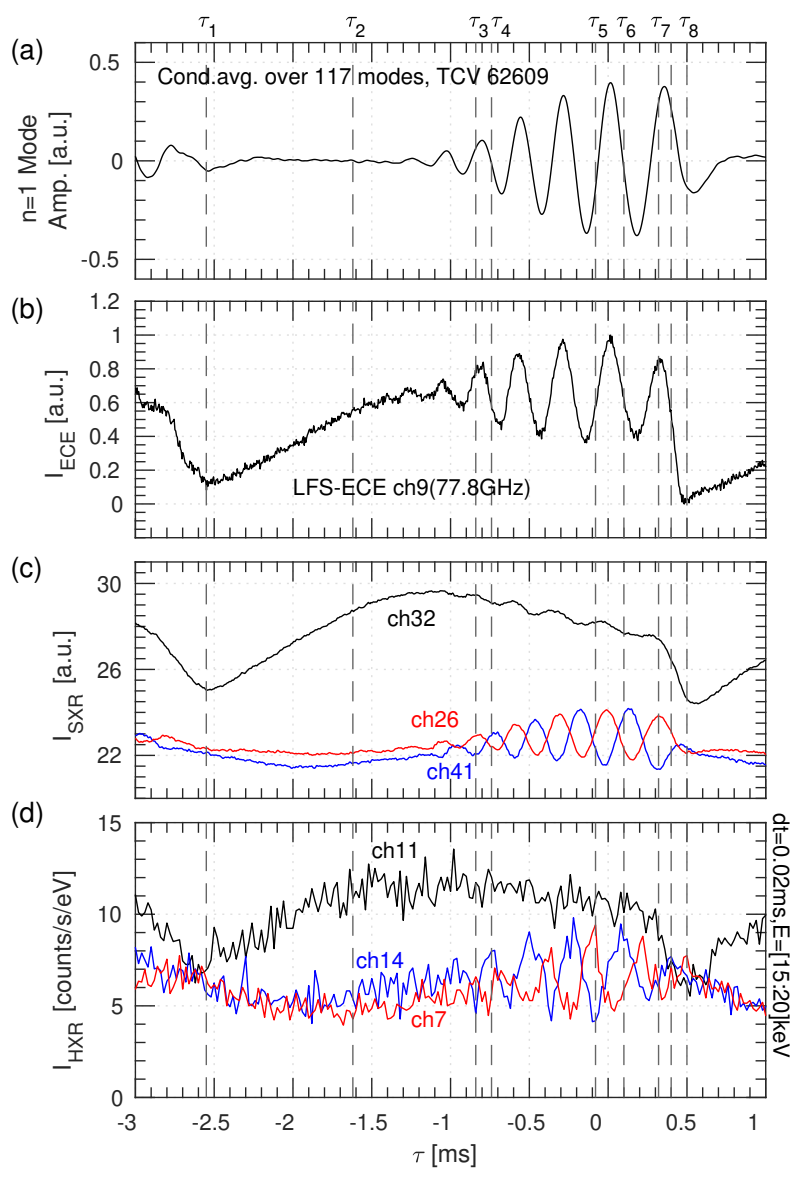

Figure 4: Conditionally averaged (a) magnetic field fluctuation, (b) thermal ECE signal, (c) soft X-ray intensity, and (d) hard X-ray count rate. Selected relative time points are indicated as dashed lines at: $\tau_{1}=-2.55 \mathrm{~ms}, \tau_{2}=-1.62 \mathrm{~ms}, \tau_{3}=-0.84 \mathrm{~ms}, \tau_{4}=-0.74 \mathrm{~ms}, \tau_{5}=$ $-0.08 \mathrm{~ms}, \tau_{6}=0.10 \mathrm{~ms}, \tau_{7}=0.32 \mathrm{~ms}, \tau_{8}=0.50 \mathrm{~ms}$.

shows the same behavior described in section 3.1 for the raw data: after $\sim 1 \mathrm{~ms}$ of the plasma ramp-up phase, $I_{S X R}$ on the central channel decreases gradually $\left(\Delta I_{S X R} \sim-2.4\right)$ during $\sim 2$ ms of fishbone-like oscillations. Then the cycle ends with a sudden crash $\left(\Delta I_{S X R} \sim-2.1\right)$ that appears between $\tau_{7}$ and $\tau_{8}$.

In the conditionally averaged hard X-ray count rate plot (Figure 4 (d)), the same behavior is observed. This analysis refers to the $15-20 \mathrm{keV}$ energy bin with an integration time of 0.02 ms. The lines of sight of the hard x-ray camera in the poloidal orientation are plotted in Figure 1 (d). The central channel (ch11) shows that the central hard X-ray signal also increases during the ramp-up phase and gradually decreases during the fishbone oscillation period. During this oscillation period, the fishbone oscillation in the hard X-ray data is observed in the channels whose line of sight is parallel to the $\mathrm{q}=1$ surface, such as ch7 and ch14. The frequency of the oscillation in $I_{H X R}$ matches the frequency of the $\mathrm{m} / \mathrm{n}=1 / 1$ oscillation, and a nearly $\pi$-phase difference is found between the two channels due to the $\mathrm{m}=1$ mode structure.

For the study of the spatial mode structure, the spatial profiles of $I_{S X R}$ and $I_{H X R}$ for the selected time points are presented in Figure 5. The Thomson scattering measurements of the electron temperature and density profiles at several relative time points $(\tau)$ are plotted in Figure 6 . The $\mathrm{q}=1$ surface locations calculated by the LIUQE equilibrium reconstruction are indicated 
(a)

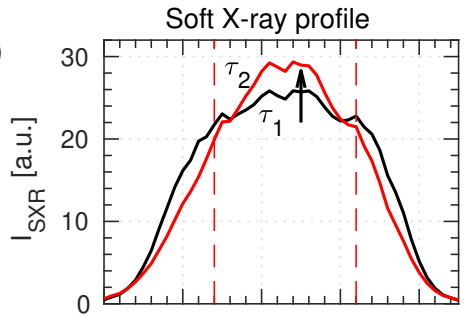

(b)

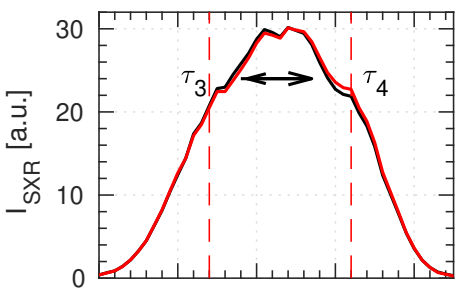

(c)

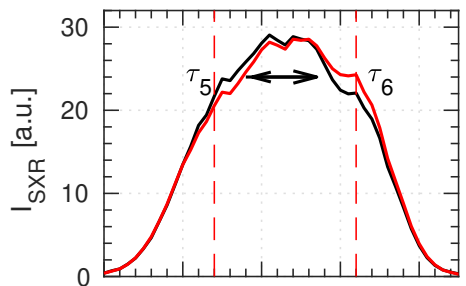

(d)

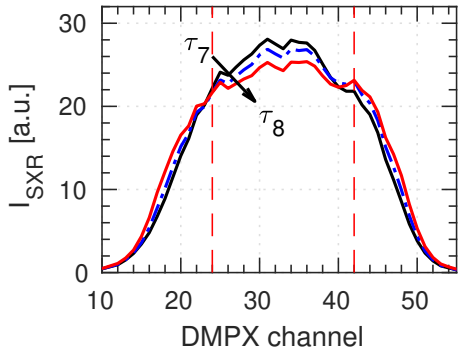

(e)

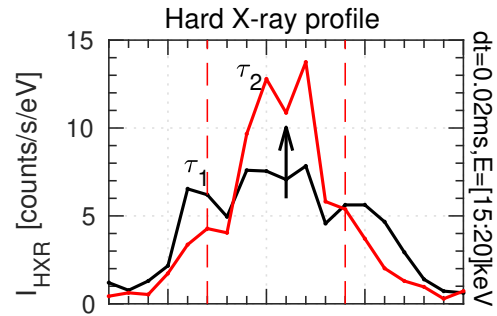

(f)

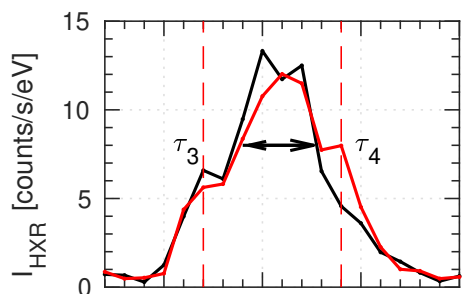

(g)

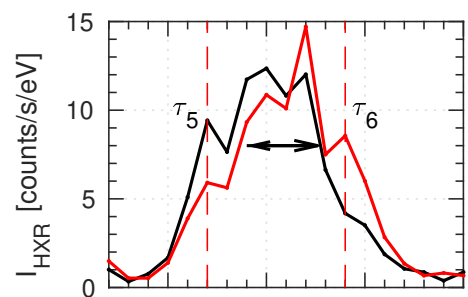

(h)

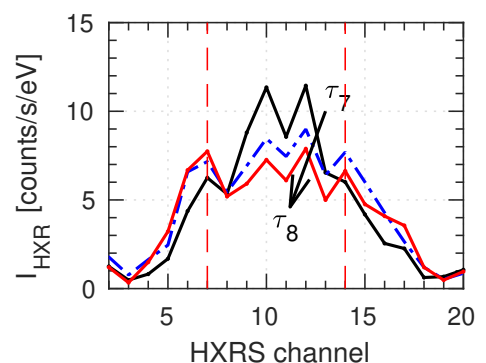

Figure 5: Soft X-ray and hard X-ray profiles at selected times, as indicated in Figure 4.
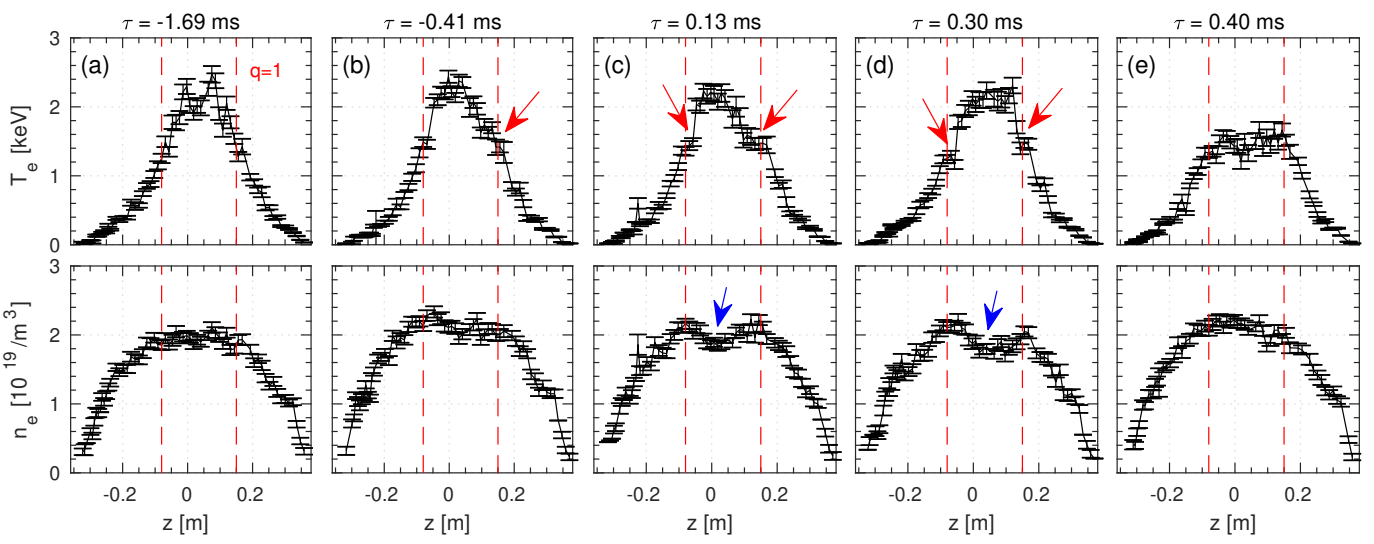

Figure 6: Electron temperature and density profiles measured by the Thomson scattering system, at selected relative times $(\tau)$ : (a) during the ramp-up phase, (b-d) during the fishbone-like oscillation, (e) after the crash. 
as red dashed lines.

During the ramp-up phase, the central intensity of both $I_{S X R}$ and $I_{H X R}$ increases (Figure $5(\mathrm{a}, \mathrm{e}))$. Profiles that are initially flat become peaked at the end of the ramp-up phase. Two peaks inside the $\mathrm{q}=1$ surface are observed due to the off-axis localized ECCD. These peaks can also be observed in the electron temperature profile (Figure 6 (a)), while the electron density profile remains constant during the ramp-up phase.

In the early phase of the $\mathrm{m}=1$ oscillation, fluctuation occurs only in a small region inside $\mathrm{q}$ $=1($ Figure $5(\mathrm{~b}, \mathrm{f}))$. Towards the end of the oscillation phase, the $I_{S X R}$ and $I_{H X R}$ profiles show a broader fluctuation region and a larger fluctuation level (Figure $5(\mathrm{c}, \mathrm{g})$ ). The localized region of fluctuation is also observed in the electron temperature profiles, indicated with red arrows in Figure 6 (b-d). This local flattening of the electron temperature is similar to the observation of the $\mathrm{m} / \mathrm{n}=1 / 1$ island in MAST [31] during the standard sawtooth crash on a much shorter time scale, for about $0.15 \mathrm{~ms}$. This observation on TCV may be also attributed to the magnetic island structure. However, due to the lack of internal magnetic field measurement, another possible explanation for this is the formation of helical core, without magnetic reconnection [32]. Further research is required to investigate the nature of the $m / n=1 / 1$ helical structure. In Figure 6 (b-d), since the rotating crescent-shaped helical structure travels past the fixed view-line of the Thomson scattering system, the size and location of the partial flattening changes.

During the oscillation phase, hollow profiles are observed in the electron density (indicated with blue arrows in Figure 6), increasingly so towards the end of the fishbone oscillation phase. In Figure 6 (d), the difference of the density in between the core and the $q=1$ surface is $19 \%$ of the density at the $\mathrm{q}=1$ surface. This is possibly due to the electron loss during the oscillation phase. The $I_{S X R}$ and $I_{H X R}$ of the central channels also show a decrease during the oscillation phase. However, since the $I_{S X R}$ and $I_{H X R}$ are line-integrated data, it is not possible to directly compare these profiles to the localized Thomson scattering measurement. The line-integrated $I_{S X R}$ and $I_{H X R}$ profiles are inverted using the Abel-inversion scheme in the next section 3.3.

At the end of the oscillation phase, in Figure $5(\mathrm{~d}, \mathrm{~h})$, the central $I_{S X R}$ and $I_{H X R}$ drop at the same time, similar to the behavior expected for a standard sawtooth crash. During the crash, both $I_{S X R}$ and $I_{H X R}$ profiles in the core decrease in amplitude and the signals of the outer channels increase, possibly because of the expelled electrons during the crash. The ECCD peaks decrease as well but remain distinctively visible. After the crash, the electron temperature inside the $\mathrm{q}=1$ surface drops and the electron density profile is no longer hollow (Figure 6 (e)).

The time evolution of $I_{H X R}$ for the higher energy bins is presented in Figure 7 (a-d) for channels 11 and 14. The fishbone-like oscillation, as well as the ramp-up and relaxation of the central $I_{H X R}$, can be observed in the lower energy range but it becomes more difficult to distinguish these from the noise as the energy increases, since the number of ECCD-accelerated electrons decreases as their energy increases. The statistics thus become insufficient to determine whether these higher-energy electrons also undergo the fishbone-like oscillation. This diagnostic limitation can be compensated by the vertical ECE diagnostic, which receives ECE from suprathermal electrons at the third harmonic, directly above the magnetic axis location at the top of the tokamak. Although the measurement is integrated in space along the vertical line, the received ECE radiation at each frequency is exclusively emitted from electrons with a specific energy, unlike the continum Bremsstrahlung radiation emitted in the hard X-ray range.

The vertical-ECE diagnostic was operational in the discharge \#62962, which is a repeat of \#62609 with higher density: the average density measured by FIR at $\mathrm{R}=0.9 \mathrm{~m}$ is up from 

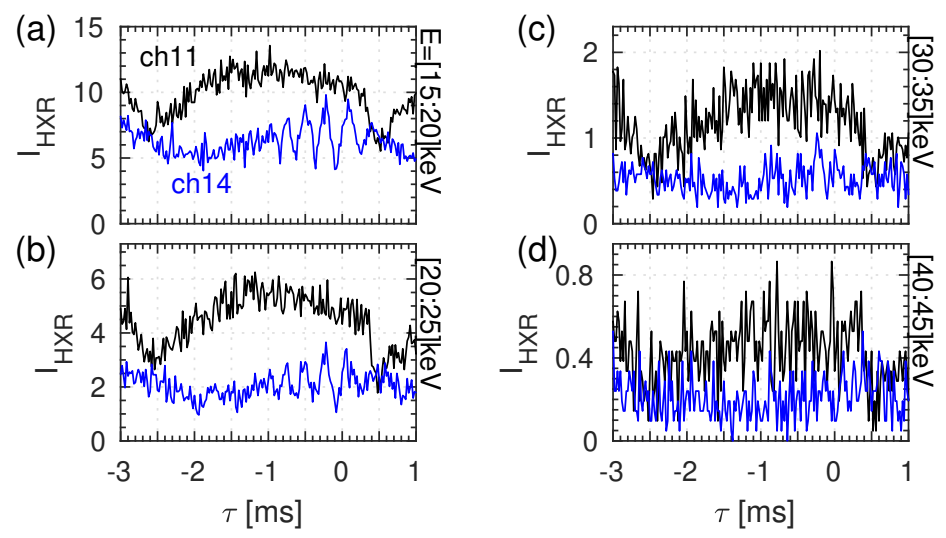

Figure 7: (left) Time evolution of the hard X-ray signal at different energy bins: (a) $\mathrm{E}=[15: 20]$ $\mathrm{keV}$, (b) $\mathrm{E}=[20: 25] \mathrm{keV}$, (c) $\mathrm{E}=[30: 35] \mathrm{keV}$, (d) $\mathrm{E}=[40: 45] \mathrm{keV}$. The time bin is $0.02 \mathrm{~ms}$.

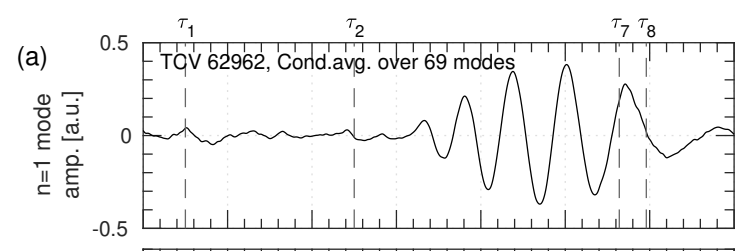

(b)

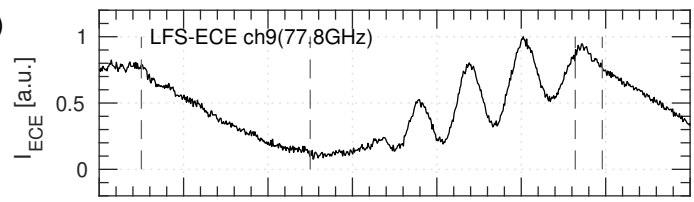

(c)

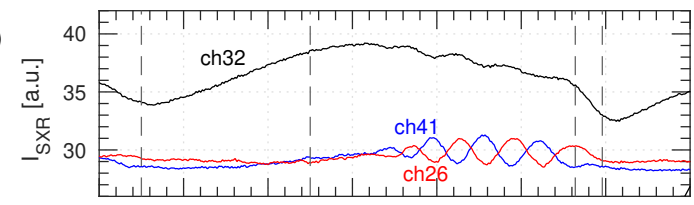

(d)

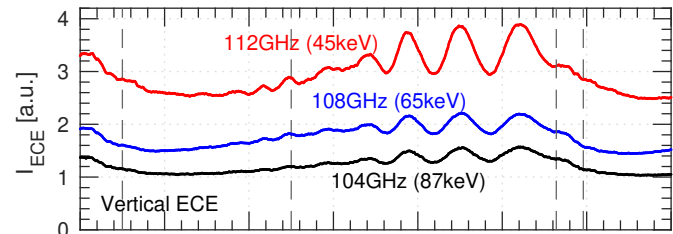

(e)

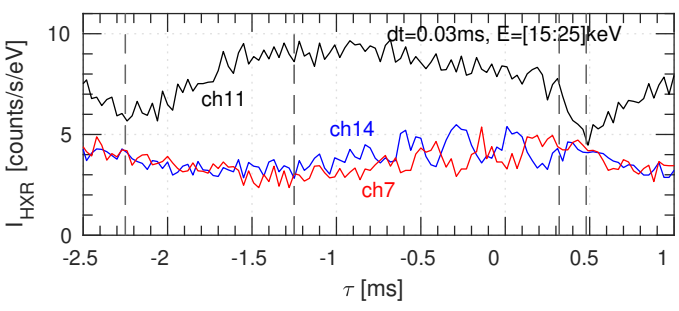

Figure 8: Conditional averaging of fishbone-like perturbations in (a) the $\mathrm{n}=1$ mode amplitude from the SVD analysis of the magnetic probe array, (b) ECE from thermal electrons, (c) soft X-ray, (d) ECE from suprathermal electrons, (e) hard X-ray count rate. Relative time points shown as dashed lines are: $\tau_{1}=-2.25 \mathrm{~ms}, \tau_{2}=-1.25 \mathrm{~ms}, \tau_{7}=0.32 \mathrm{~ms}, \tau_{8}=0.48 \mathrm{~ms}$. 
$1.78 \times 10^{19} / \mathrm{m}^{3}$ to $1.99 \times 10^{19} / \mathrm{m}^{3}$. The LIUQE equilibrium reconstruction shows that the $\mathrm{q}=1$ surface is located at the same location as \#62609. In Figure 8, conditionally averaged fishbonelike oscillations observed in several diagnostics are presented for the discharge \#62962, similarly to the data shown in Figure 4. In this discharge, the electron fishbone lasted $900 \mathrm{~ms}$, which is shorter than in \#62609 (1400 ms), reducing the statistics. Also because of the increased electron density, the hard X-ray count rate is lower than for \#62609. Therefore the time bin length for the conditional averaging is larger in \#62962 than \#62609 (0.03 ms compared to $0.02 \mathrm{~ms}$ ), as presented in Figure 8 (e). The fishbone-like oscillation is also observed in the several channels of the vertical ECE system, which correspond to different energetic electron energy ranges, as indicated in Figure 8 (d). This shows that electrons up to $\sim 100 \mathrm{keV}$ exhibit the fluctuation as observed in other diagnostics.

\subsection{Abel-inversion of the soft X-ray and hard X-ray profiles}

In order to study the redistribution of electrons over flux surfaces, the line-integrated soft X-ray and hard X-ray measurements need to be inverted. In both $I_{S X R}$ and $I_{H X R}$ measurements presented in section 3.2, no lines of sight cross one another because only a single camera is used in each measurement to receive the line-integrated signals. In this case Abel-inversion can be used to invert the data [33], with the assumption of uniform emissivity of $I_{S X R}$ or $I_{H X R}$ over the poloidal angle at each flux surface.

When the hard X-ray lines of sight cover the poloidal plane, trapped electrons are the main contributors to the detected hard X-rays, due to their preferred radiation pattern towards the propagation direction [26]. In the discharge \#62609, the bounce frequency of trapped electrons (at $\left|p_{\|} / p\right|_{\theta=0}=0.2$ ) near the $\mathrm{q}=1$ surface was $2.2 \mathrm{MHz}$ for the thermal electrons at $2.4 \mathrm{keV}$, and $6.4 \mathrm{MHz}$ and $14.4 \mathrm{MHz}$ for the suprathermal electrons at $20 \mathrm{keV}$ and $100 \mathrm{keV}$, respectively. Thus we may assume that we measure the orbit-averaged hard X-ray emission.

In fact the hard X-ray emission in the poloidal plane exhibits an asymmetry from high field side to low field side due to conservation of the magnetic moment over a collisionless particle orbit, which reduces the parallel energy on the high field side [34]. Another possiblity that can give rise to an asymmetry is the helicity of the magnetic field, but in tokamak plasmas, this effect is negligible [35]. A previous study of these effects, using a vertically viewing hard X-ray camera and using Fokker-Planck modeling, showed that the assumption of poloidal symmetry in the hard X-ray emission for Abel-inversion is justified except in extremely off-axis RF deposition cases [35]. This effect is studied for this discharge as well in section 5.1, in the Fokker-Planck modeling of this discharge.

Since the emission is assumed to be uniform at each flux surface, it is important to have an appropriate magnetic equilibrium that reflects the observed mode structure. In section 3.2, during the fishbone oscillation phase, localized flattening of the electron temperature profile is observed (Figure 6), due to the helical mode structure. As the rotating crescent-shaped mode moves past the view-line of the Thomson scattering system, the width of the locally flattened temperature region changes. This observation has been reflected in the modeling of a deformed magnetic equilibrium profile with accurate phasing. Figure 9 (a) shows the original equilibrium reconstructed by the code LIUQE and Figure 9 (b) shows an example of deformed equilibrium. In Figure 9 (b), the crescent-shaped structure is positioned at $\rho_{\psi}=0.26$ (red line) and the $\mathrm{x}$-point is rotated by $261^{\circ}$ from the low field side, in order to match the width and the phase of the oscillation in the measured hard X-ray profile in Figure 9 (c). The flux surfaces inside 

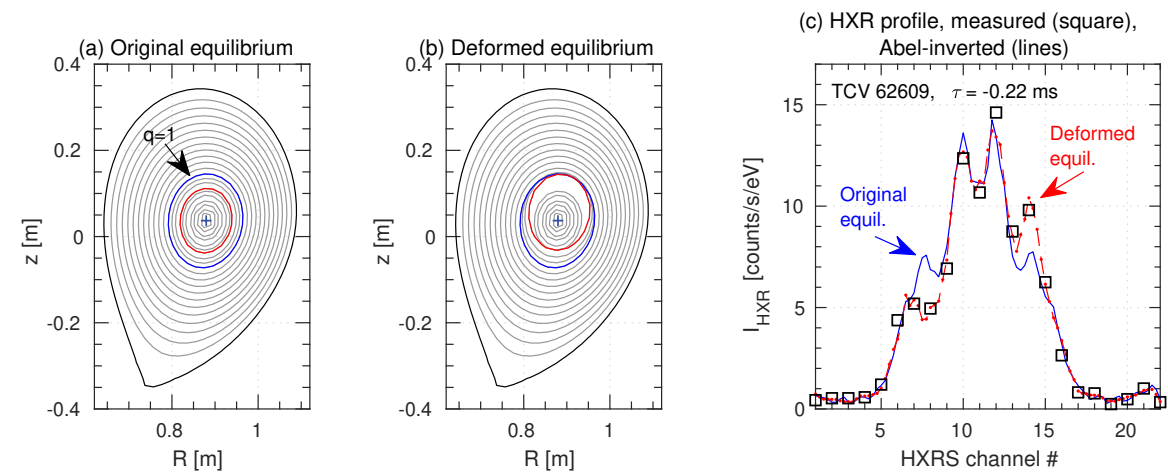

Figure 9: (a) Equilibrium reconstructed by LIUQE, (b) deformed equilibrium model used in the Abel-inversion. (c) Experimental hard X-ray profile (square) and reconstructed profiles obtained by line-integrating the Abel-inverted profiles, using the default equilibrium model (blue solid line) and the helical equilibrium model (red dashed line).

(1)

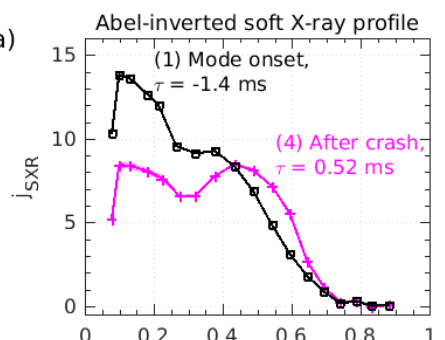

(b)

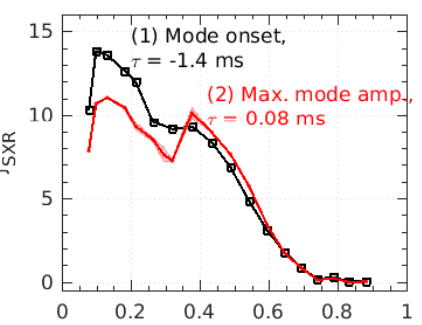

(c)

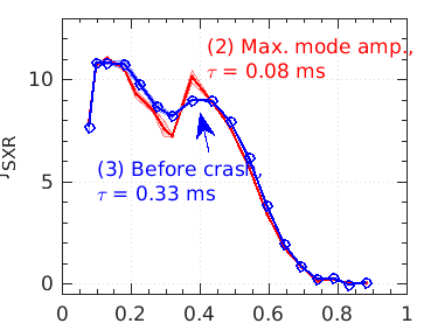

(d)

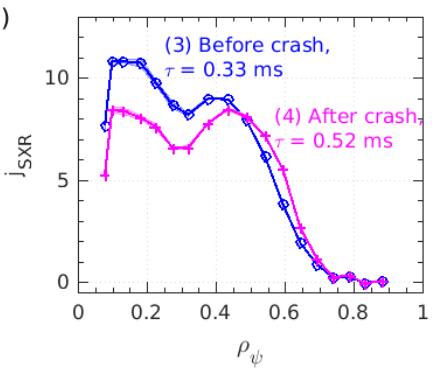

(e)

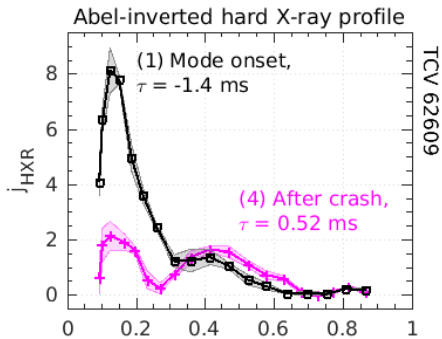

(f)

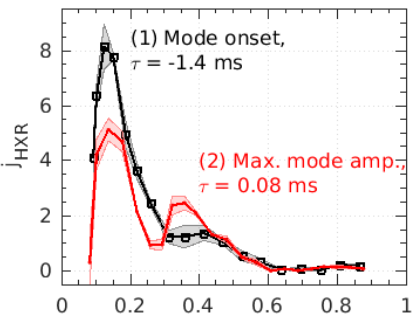

(g)

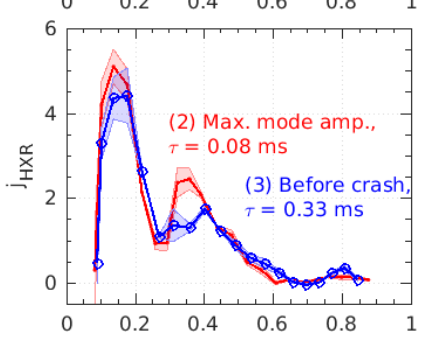

(h)

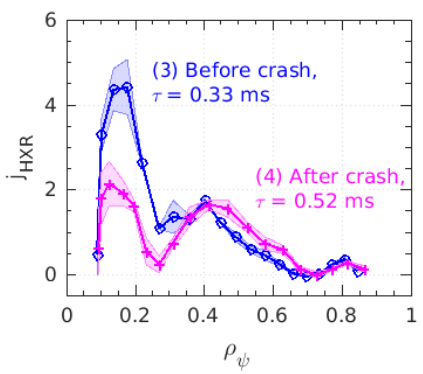

Figure 10: Abel-inverted soft X-ray and hard X-ray profiles for selected time points. 
$\rho_{\psi}=0.26$ are unchanged.

In order to assess the inversion result, the line-integrated $I_{H X R}$ data are reconstructed from the Abel-inverted hard X-ray profiles, in Figure 9 (c). It is clear that both models can reconstruct the symmetric signals near the magnetic axis. However, in the fluctuation region near the $\mathrm{q}=1$ surface, near channels 7 and 14, the experimental hard X-ray profile can be reconstructed only using the deformed equilibrium. The root-mean-square errors are 0.97 and 0.44 , for the original equilibrium and deformed equilibrium, respectively. In this reconstruction, 22 flux surfaces are included in the equilibrium. Since the hard X-ray camera has fewer measurement points (24 channels in total) than the soft X-ray measurement (64 channels in total), virtual lines of sight are added in between real lines of sight, and the measured data are interpolated on these virtual channels. A least-squares method is used to find a solution.

Figure 10 presents the Abel-inverted soft X-ray and hard X-ray profiles, $j_{S X R}$ and $j_{H X R}$, for four selected time poins: (1) at the onset of the oscillation, which is at the end of the ramp-up phase, (2) in the middle of the fishbone oscillation phase, around the maximum of the oscillation amplitude, (3) at the end of the oscllation phase, right before the sudden crash, (4) after the sudden crash. The data are plotted for pairs of adjacent time points, to allow direct comparisons and an assessment of the time evolution. The relative time $\tau$ is identical to that given in Figure 4.

During the ramp-up phase, the ECCD peaks in $j_{S X R}$ and $j_{H X R}$ near $\rho_{\psi} \sim 0.1$ increase (Figure $10(\mathrm{a}, \mathrm{e})$ ). The intensity outside $\rho_{\psi} \sim 0.4$ decreases as the electrons outside the ECCD deposition area, which accumulated during the previous crash, are lost. Since the $m / n=1 / 1$ mode grows at this point, (1), the fishbone stability study in section 5 will be based on the electron distribution function of this point.

During the first half of the fishbone oscillation phase, the ECCD peak in both $j_{S X R}$ and $j_{H X R}$ decreases in Figure $10(\mathrm{~b}, \mathrm{f})$. The outer peak increases at $\rho_{\psi} \sim 0.36$, with the change in $j_{H X R}(99 \%)$ more significant than that of $j_{S X R}(13 \%)$. In the oscillation phase, the Abelinversion is based on the deformed equilibrium model, as presented in Figure 9. The helical mode structure is positioned at $\rho_{\psi}=0.26$, between the two peaks. This shows that the core population decreases during the mode rotation, and the expelled electrons accumulate outside the rotating helical structure. The radial displacement of the electrons can be related to the energy loss of the electrons as a result of a resonant interaction with the mode [36]. In section 5, the possibility of the resonant destabilization will be demonstrated by solving a linear fishbone dispersion relation. A further simulation during the non-linear phase is needed in the future in order to investigate the redistribution of the electrons.

During the second half of the fishbone oscillation phase (Figure $10(\mathrm{c}, \mathrm{g})$ ), there is no significant change in the ECCD peaks, but the other peak at $\rho_{\psi} \sim 0.36$ is reduced to the initial level of (1). This may be explained by the decreased ECCD peak at (2), compared to (1): the effective electron source is smaller than in (1), thus the loss of the accumulated electrons at $\rho_{\psi} \sim 0.36$ can no longer be fully compensated. This suprathermal loss outside the mode structure can be attributed to either collisional slowing down or anomalous transport [37,38], depending on the level of turbulent driven fluctuations.

The end of the oscillation is followed by the sudden loss of the central pressure, as indicated in Figure $10(\mathrm{~d}, \mathrm{~h})$. The ECCD peaks in both $j_{S X R}$ and $j_{H X R}$ decrease significantly but the central value $\left(\rho_{\psi} \sim 0\right)$ of $j_{S X R}$ does not drop to zero because of the thermal electron contribution. In $j_{H X R}$, the central value does drop to nearly zero but the ECCD peak still exists, because the EC 
(a)

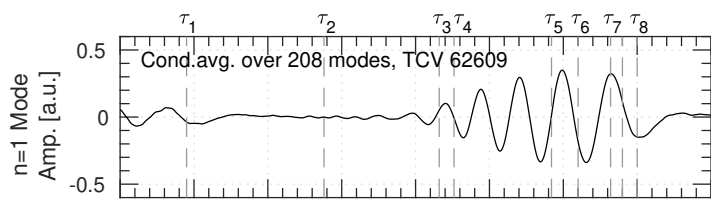

(b)

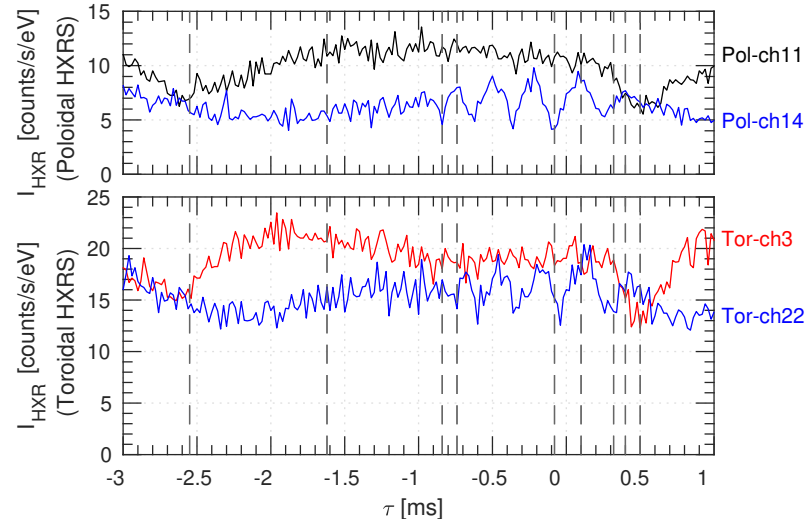

Figure 11: Conditional averaging results of (a) magnetic field perturbation, (b) hard X-ray signals in channels 11 and 14 in the poloidal orientation, (c) hard X-ray signals in channels 3 and 22 in the toroidal orientation. In the hard X-ray analysis, $\mathrm{dt}=0.02 \mathrm{~ms}$ and $\mathrm{E}=[15: 20]$ $\mathrm{keV}$. The relative time points from $\tau_{1}$ to $\tau_{8}$ are identical to those in Figure 4.

wave is constantly applied during the discharge. The other peak outside $\rho_{\psi} \sim 0.26$ still remains, due to the loss of the central electrons.

\subsection{The different response of passing and trapped electrons}

Since suprathermal electrons emit Bremsstrahlung radiation preferentially in the direction of their propagation, in the hard X-ray measurement with a poloidally oriented camera, forward emission of the bouncing trapped electrons is the main contributor to the collected hard Xray photons, along with the perpendicular Bremsstrahlung emission of passing electrons. As presented in Figure 1 (c), the TCV hard X-ray camera can be rotated toroidally so some lines of sight can be parallel to the toroidal magnetic field lines. The TCV discharge \#62609 analyzed so far was repeated with the hard X-ray camera in the toroidal orientation, as shown in Figure 11 (c). In this case forward Bremsstrahlung emission of passing electrons is the main contributor to the photon detection of channel 3 (Tor-ch3), as well as perpendicular emission of trapped electrons. Since the intensity of backward Bremsstrahlung emission is negligible compared to that of forward or perpendicular emission, the perpendicular emission of trapped electrons is expected to be dominant in the photon detection of channel 22 (Tor-ch22).

During the fishbone oscillation phase, $I_{H X R}$ of Tor-ch3 stays relatively constant while Torch22 exhibits a fishbone oscillation as observed in the poloidal orientation (Figure 11 (b)). The constant signal observed in Tor-ch3 can be attributed to the fact that there is no significant loss of passing electrons and no accumulation of passing electrons outside the helical structure. If passing electrons were also expelled during this phase, $I_{H X R}$ would have shown even stronger fluctuation than that of Tor-ch22. This is possibly due to the highly constrained conditions for effective passing electron resonant interaction [39] (see also section 5.2). 


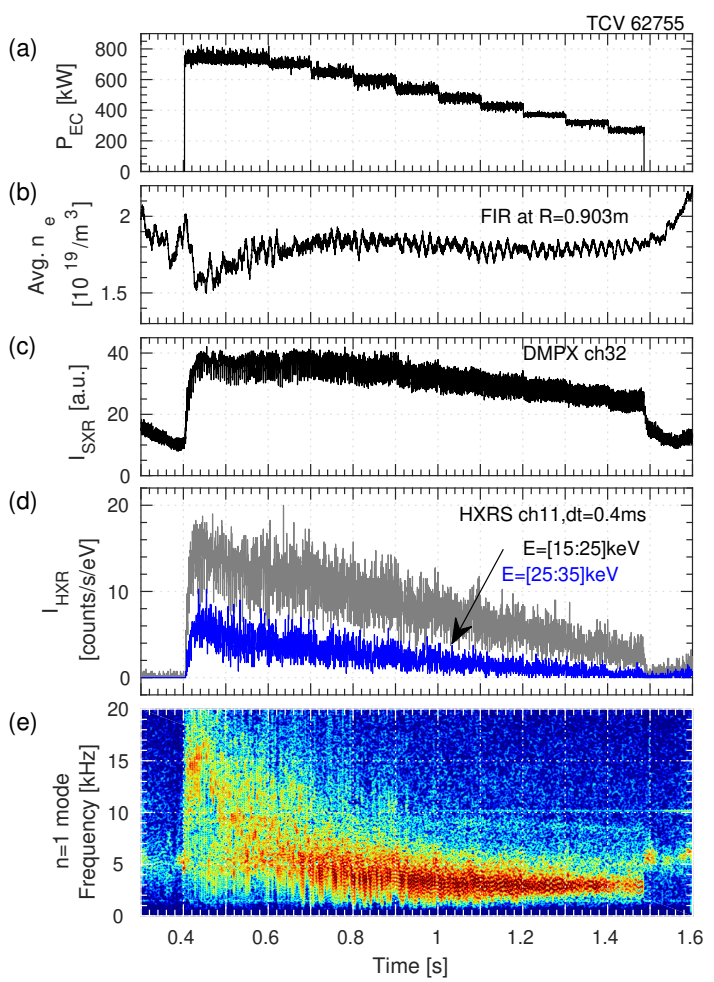

Figure 12: Time evolution of plasma parameters in \#62755: (a) ECCD power, (b) line-averaged electron density, (c) central soft X-ray, (d) hard X-ray count rate, (e) spectrogram of the $\mathrm{n}=1$ mode detected by the toroidal magnetic probe array.

\section{The effect of ECCD parameters on the onset of the fish- bone instability}

In the previous section, we have seen that during the fishbone oscillation phase, core electrons are expelled and the mode frequency chirps down. The non-linear growth of the $\mathrm{m} / \mathrm{n}=1 / 1$ mode can be closely related to the redistribution of the suprathermal electrons. In this section, we study the behavior of the fishbone oscillation in globally varying experimental conditions: we present results from an ECCD power scan in section 4.1, and from a magnetic field scan in section 4.2 .

\subsection{ECCD power scan}

Based on the discharge \#62609 analyzed in the previous section, the ECCD power was scanned in \#62755, as shown in Figure 12 (a). The EC power of $750 \mathrm{~kW}$ is turned on at $0.4 \mathrm{~s}$, then from $0.6 \mathrm{~s}$ at each $0.1 \mathrm{~s}$, the power is decreased by $50 \mathrm{~kW}$, keeping the injection angle fixed. The power transition at each step takes about $4 \mathrm{~ms}$. The plasma current is kept constant at $210 \mathrm{kA}$ and the LIUQE equilibrium reconstruction shows that the $\mathrm{q}=1$ surface is located at $\rho_{\psi}=0.48$ \pm 0.01 throughout the discharge. The toroidal magnetic field is kept constant at $1.34 \mathrm{~T}$ from $0.5 \mathrm{~s}$. The plasma toroidal rotation frequency near the $\mathrm{q}=1$ surface is measured to be $4.1 \pm$ $0.8 \mathrm{kHz}$ during ECCD, in the plasma current direction. The directions of the plasma current, toroidal magnetic field, and toroidal plasma rotation are identical as indicated in Figure 1 (a). 

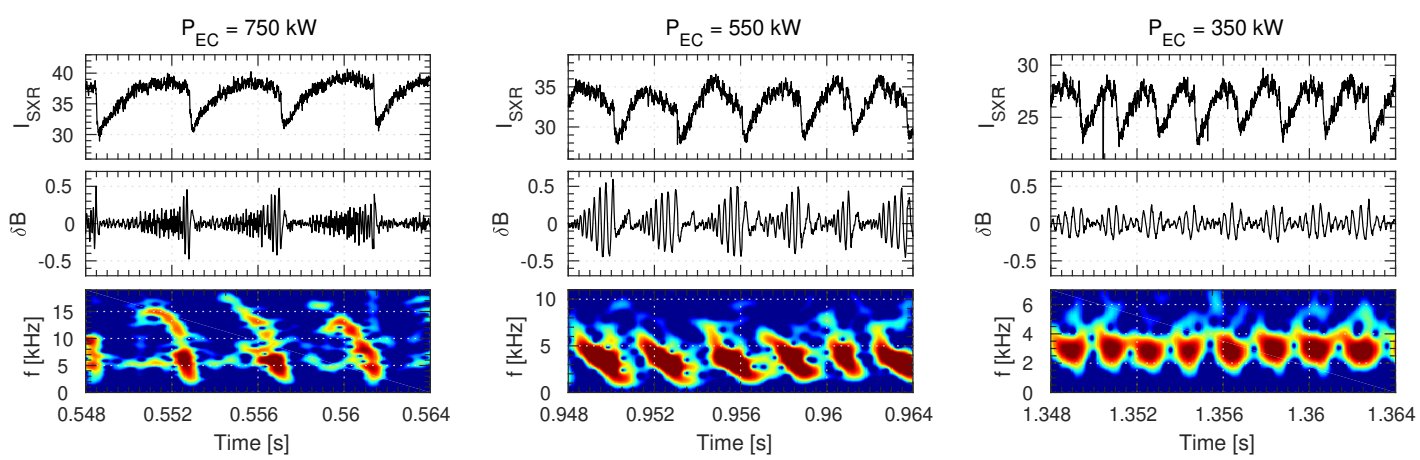

Figure 13: Soft X-ray signal $I_{S X R}$ of channel 32 , the $\mathrm{n}=1$ mode amplitude in the magnetic probe $(\delta B)$, and Fourier spectrogram of $\delta B$ are plotted.

The suprathermal electron population can be detected in the hard X-ray camera, as shown in Figure 12 (d). The suprathermal population decreases as the EC power decreases. The hard X-ray camera was in the poloidal orientation, so the line of sight of channel 11 goes through the magnetic axis.

In this discharge, the $\mathrm{m} / \mathrm{n}=1 / 1$ mode oscillation is also observed near the $\mathrm{q}=1$ surface during the ECCD phase. Figure 12 (e) shows the spectrogram of the $\mathrm{n}=1$ mode, obtained from the SVD analysis of the magnetic probe data from the toroidal array. As the EC power is turned on, the mode appears with relatively high frequency in the beginning, and the mode frequency decreases as the ECCD power decreases. The amplitude of the mode oscillation, which can be seen from the power of the Fourier spectrum indicated in color (blue and red correspond to low and high spectral power, respectively), shows that the mode amplitude also changes over time, but does not decrease monotonically as the frequency does: the mode amplitude has a maximum value at $\sim 1.0 \mathrm{~s}$.

The $\mathrm{m} / \mathrm{n}=1 / 1$ perturbation and the spectrogram are presented in detail in Figure 13, for three selected time windows with different EC powers. In all time windows, the fishbonelike bursts are accompanied by the non-standard sawtooth $\left(I_{S X R}\right.$ of DMPX ch32). The mode frequency is confirmed to decrease, following the decrease in EC power from $750 \mathrm{~kW}$ to $350 \mathrm{~kW}$, from the first to the third column. In the first column, distinctive sawtooth precursors at $\sim 5$ $\mathrm{kHz}$ are observed at the end of the non-standard cycle. This supports the conclusion that the observed fishbone modes are distinct from the standard sawtooth precursors, which have the same $\mathrm{m} / \mathrm{n}=1 / 1$ mode structure. The stability properties of this discharge at varying EC power are assessed in section 5 by solving the linear fishbone dispersion relation.

The period of the non-standard sawtooth cycle decreases with diminishing ECCD power. As analyzed in section 3, the non-standard sawtooth period is directly related to the fishbone oscillation duration: after the ramp-up phase, the central $I_{S X R}$ saturates or decreases during the mode oscillation, then the cycle ends with a sudden crash at the end of the fishbone oscillation. The suprathermal electron population, which depends on the EC power level, destabilizes the mode but at the same time is ejected by interacting with the mode. Therefore a balance between the acceleration and loss of suprathermal electrons determines the fishbone period, as for ion fishbones [40]. A further analysis that considers the non-linear growth of the fishbone mode is needed to predict the mode cycle. 

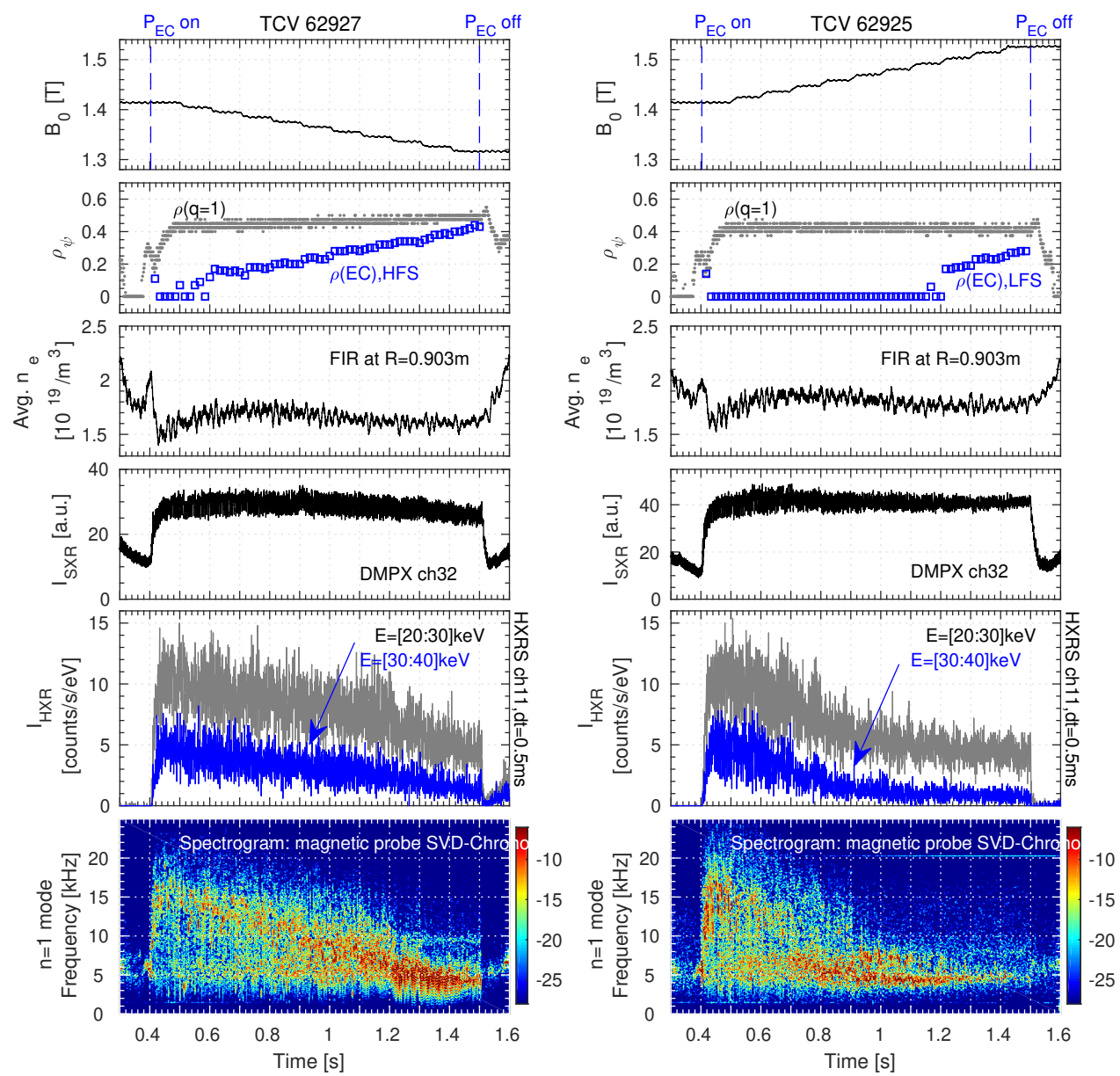

Figure 14: Toroidal magnetic field ramp-down discharge \#62927 (left) and ramp-up discharge \#62925 (right). From top to bottom, each row corresponds to: toroidal magnetic field, the $\mathrm{q}=$ 1 surface location and the peak $P_{E C}$ location, line-averaged electron density, central soft X-ray intensity, hard X-ray count rates from different energy bins, and the spectrogram of the $\mathrm{n}=1$ mode oscillation.

\subsection{Magnetic field scan}

In order to study the effect of the ECCD location on the behavior of the $\mathrm{m} / \mathrm{n}=1 / 1$ mode oscillation, we have scanned the localized ECCD $(750 \mathrm{~kW})$ deposition location from the high field side to the low field side, by varying the toroidal magnetic field, as presented in Figure 14. In the discharge \#62927, the magnetic field intensity was ramped down from $1.42 \mathrm{~T}$ to $1.32 \mathrm{~T}$ by $0.01 \mathrm{~T}$ at every $0.1 \mathrm{~s}$, thus the ECCD location is moved towards the high field side. The ray tracing calculation shows that the absorbed EC power $\left(P_{E C}\right)$ has a gaussian shape and the full width half maximum (FWHM) is $\sim 0.25$. In Figure 14 the localized ECCD location corresponds to the peak position of the EC power profile. As the ECCD location moves out, the suprathermal population and the mode frequency decrease. This relation between the suprathermal population and the mode behavior is similar to the result obtained in the previous section during the ECCD power scan.

In the discharge \#62925, the magnetic field was ramped up from $1.42 \mathrm{~T}$ to $1.52 \mathrm{~T}$ by $0.01 \mathrm{~T}$ at every $0.1 \mathrm{~s}$, thus the ECCD location moves towards the low field side. As one can see in Figure 

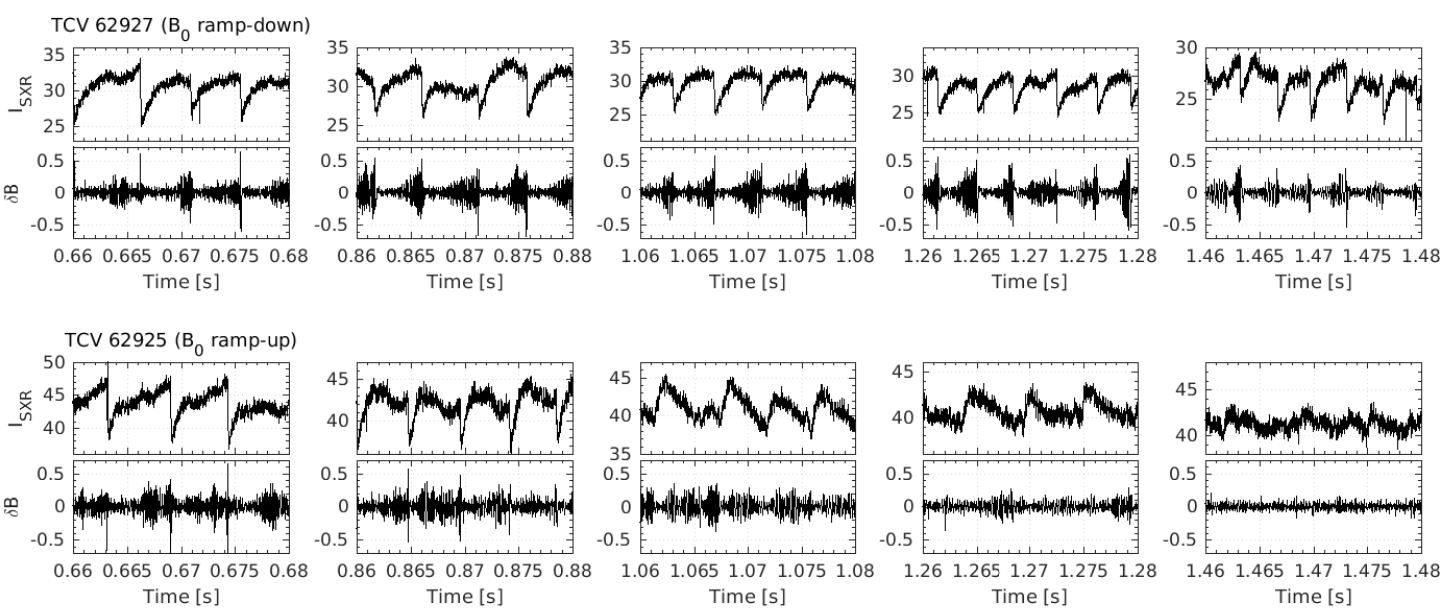

Figure 15: Central soft X-ray intensity $I_{S X R}$, from DMPX channel 32, and the $\mathrm{n}=1$ mode perturbation from the SVD analysis of the toroidal magnetic probe array, for selected time windows of \#62927, B-field ramp-down discharge, and \#62925, B-field ramp-up discharge.

16, the EC resonance layer passes the magnetic axis, thus the EC power peak is concentrated at the magnetic axis from $\sim 0.4$ s to $\sim 1.2 \mathrm{~s}$. Nevertheless, it should be kept in mind that the EC resonance layer shifts, as can be seen from the changing width of $P_{E C}$ in Figure 16 . The absolute level of $I_{S X R}$ changes compared to \#62927 because the measurement gain is different in the two discharges. The hard X-ray data shows that the suprathermal electron population also decreases, in the same way as in the B-field ramp-down case, but at a faster rate: $I_{H X R}$ of $\mathrm{E}$ $=[20: 30] \mathrm{keV}$ drops down to 5 at only $0.8 \mathrm{~s}$ when the ECCD is still localized near the magnetic axis, while in \#62927 $I_{H X R}$ reaches 5 at $1.4 \mathrm{~s}$, when ECCD is localized at $\rho_{\psi} \sim 0.4$. As the suprathermal electron population decreases, the mode frequency also decreases as observed in the B-field ramp-down discharge, and the mode almost disappears after $1.3 \mathrm{~s}$.
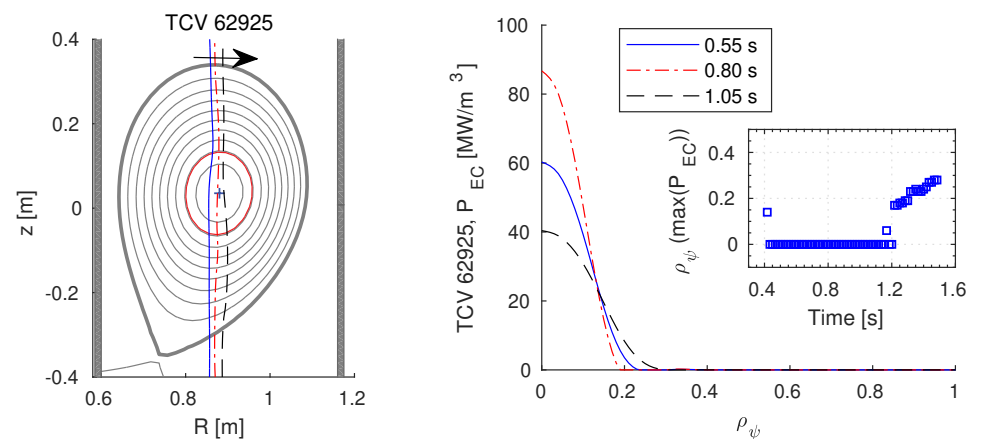

Figure 16: (Left) EC resonance layer shifts and (right) absorbed EC power profiles at selected times of discharge \# 62925 .

The changing mode behavior, as well as the ECCD location scan, has an effect on the central plasma relaxation, as presented in Figure 15. In the high field side ECCD case ( $B_{0}$ rampdown), the central plasma relaxation keeps the saturated-sawtooth shape [29], and the standard sawtooth precursor is often separated from the fishbone-like perturbation. In the low field side ECCD case ( $B_{0}$ ramp-up), the saturated shape transitions to an inverted shape, and then 
no significant central plasma relaxation is found when the ECCD is located at the outermost location.
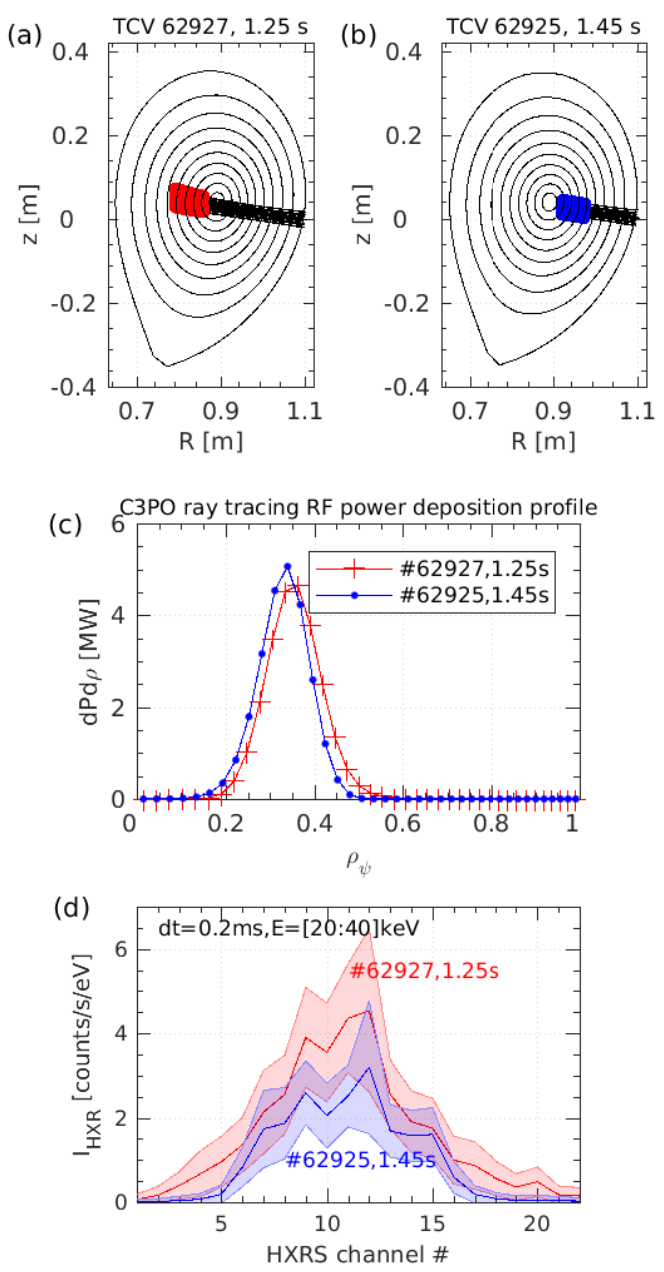

Figure 17: Poloidal ray tracing trajectories of (a) high field side ECCD and (b) low field side ECCD. (c) The RF power deposition profiles. (d) Averaged hard X-ray count rate profiles for the two time points shown in (a) and (b).

In Figure 17, an example of the asymmetric suprathermal electron distribution is presented for the low field side and the high field side ECCD cases. At $1.25 \mathrm{~s}$ of \#62927 (Figure 17 (a)), the ECCD is localized at $\rho_{\psi} \sim 0.35$ on the high field side and at $1.45 \mathrm{~s}$ of \#62925 (Figure 17 (b)), the ECCD is localized also at $\rho_{\psi} \sim 0.35$ but on the low field side. In Figure 17 (d), the hard $\mathrm{X}$-ray profiles collected at every $0.2 \mathrm{~ms}$ are averaged at $1.25 \pm 0.5 \mathrm{~s}$ in \#62927 and at $1.45 \pm 0.5$ $\mathrm{s}$ in \#62925, in the energy bin of $\mathrm{E}=[20: 40] \mathrm{keV}$. The two profiles show similar peak positions near hard X-ray channels 9 and 12, because the ECCD is localized at $\rho_{\psi} \sim 0.35$ in both cases. However, the core $I_{H X R}$ from the high field side ECCD is higher than that from the low field side ECCD. This can be attributed to the electron trapping effect, which decreases the ECCD efficiency due to the fast bounce motion of the trapped electron fraction [41-43], which hinders electrons from absorbing energy from the EC beam. This effect is stronger on the low field side because of the higher trapped electron fraction. In addition, the $I_{H X R}$ profile of the high field side case is broader than that of the low field side, as seen on the channels outside 7 and 15 . 
This can be due to the stronger $\mathrm{m} / \mathrm{n}=1 / 1$ mode activity in the high field side ECCD case, which regularly expels electrons, as observed in section 3 .

\section{Numerical analysis of the $m / n=1 / 1$ mode}

In order to assess the stability of the fishbone mode during the ECCD discharges in TCV, the plasma is modelled using the three-dimensional relativistic bounce-averaged Fokker-Planck solver LUKE [44], with the aid of a hard X-ray synthetic diagnostic [35], in section 5.1. The electron distribution function is used to solve the linear fishbone dispersion relation using the code MIKE [45], in section 5.2. The effect of the electron distribution function change on the mode behavior is studied as well.

\subsection{Fokker-Planck modeling of the experiment}

Fokker-Planck modeling of the plasma has been performed at the mode onset of the discharge $\# 62609$, which corresponds to the relative time point $\tau_{2}$ in Figure 4 at the end of the ramp-up phase, when the $\mathrm{m} / \mathrm{n}=1 / 1$ mode oscillation starts to grow. The electron temperature and density profiles from the Thomson scattering system, the plasma equilibrium structure from the LIUQE equilibrium reconstruction, the effective charge from the CXRS diagnostic, and the loop voltage measurement are used as input data in the Fokker-Planck solver LUKE [44]. The ray-tracing code C3PO [28] is coupled to LUKE to model the EC wave propagation, and the modelled ray properties are used to build the quasilinear RF diffusion coefficient in the FokkerPlanck calculation. The simulation time and suprathermal electron transport terms are used as free parameters, so the hard X-ray synthetic diagnostic result matches both the amplitude and width of the hard X-ray measurement.

Unlike other Fokker-Planck studies on ECCD discharges where the steady-state solution is considered, a finite Fokker-Planck calculation time is applied in this modeling. If the plasma can be described as a Maxwellian after the collapse, we can apply the ramp-up duration as a simulation time and calculate the electron distribution function from the Maxwellian distribution determined by the given temperature. However, the hard X-ray measurements presented in section 2 have shown that the plasma after the crash is in fact not in a Maxwellian state due to the significant fraction of suprathermal electrons remaining after the crash. Therefore the simulation time is adjusted to be longer than the ramp-up period and is set to $\tau_{F P}=9.6 \mathrm{~ms}$. This is found to be enough to create high energy electrons so the synthetic diagnostic can match the experimental hard X-ray measurement.

The suprathermal electron transport term is left as a free parameter, which plays an important role in modeling the ECCD plasma $[46,47]$. The choice of an appropriate transport term is aided by the hard X-ray synthetic diagnostic, using the Fast Electron Bremsstrahlung (FEB) module in the LUKE code [35]. It calculates the Bremsstrahlung radiation from the electron distribution considering the magnetic equilibrium, and the photons collected at each detector are calculated by integration along their line of sight for the specifc geometry of the diagnostic. Finally, the response function of the CdTe detector is used to transform the received photon spectrum to the pulse spectrum, which can be directly compared to the experimentally measured hard X-ray data.

When no suprathermal electron transport is assumed ( $T_{0}$ in Figure 18 (a)), the reconstructed hard X-ray profiles have two localized ECCD peaks that are larger in absolute value than the 


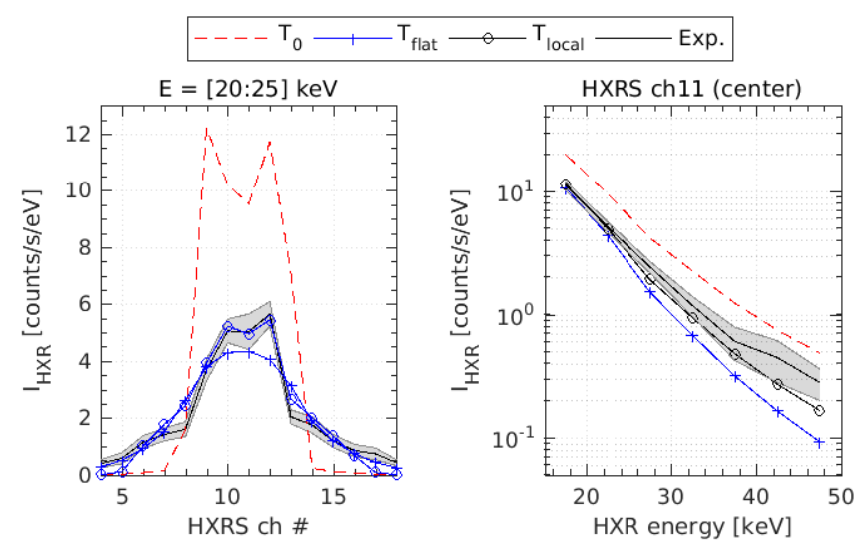

Figure 18: (a) Line-integrated hard X-ray emissivity (count rate) profile from the experimental measurement and from LUKE-FEB, using no transport $\left(T_{0}\right)$, spatially flat transport $\left(T_{\text {flat }}\right)$, localized transport near the helical mode struture $\left(T_{\text {local }}\right)$. (b) Energy spectrum of $I_{H X R}$ from the experiment and simulations using different transport models.

measured $I_{H X R}$. The calculated plasma current $I_{p, T_{0}}=-230 \mathrm{kA}$ is also larger than the experimental value $I_{p, \exp }=-211 \mathrm{kA}$. The broad $I_{H X R}$ profile outside the ECCD area (outside HXRS channels 7 and 14) is not reproduced by the simulation, due to the transport of the suprathermals. The broad $I_{H X R}$ profiles are reconstructed when we introduce a spatially flat transport model $\left(T_{\text {flat }}\right.$ in Figure 18 (a)). The radial diffusion parameter is set to $D_{r 0}=1.5 \mathrm{~m}^{2} / \mathrm{s}$, so the calculated $I_{p, T_{f l a t}}=-211 \mathrm{kA}$ also matches the experimental value. However, in this case the transport term smoothes out the localized ECCD peaks, such that the core $I_{H X R}$ profiles cannot be reproduced. In addition, the central $I_{H X R}$ shows that the high energy electrons are predicted to be lost more rapidly than in reality, see Figure 18 (b).

The Abel-inversion of the soft X-ray and hard X-ray profiles in section 3.3 showed that electron transport is enhanced near the helical mode structure during the fishbone oscillation and the sawtooth-like crash. At the onset of the mode, the electrons expelled before the ramp-up phase are still not lost, as experimentally observed. Thus a transport channel that is localized at the location of the helical mode structure is implemented. The radial diffusion coefficient is modelled as a Gaussian peak, $D_{r}\left(\rho_{\psi}\right)=D_{r 0} \exp \left(-\left(\rho_{\psi}-\rho_{0}\right) / 2 / \sigma^{2}\right)$, with $D_{r 0}=5.0, \rho_{0}=0.28$, and $\sigma=0.06 . \rho_{0}$ and $\sigma$ are determined from the transport channel around $\mathrm{q}=1$, which was observed in Figure 10. Then the height of the Gaussian $D_{r 0}$ is adjusted, so the calculated $I_{p, T_{l o c a l}}$ $=-211 \mathrm{kA}$ also matches the experimental value. The reconstructed hard X-ray profile using this model ( $T_{\text {local }}$ in Figure 18 (a)) matches well the experimental $I_{H X R}$, both ECCD peaks and the broad profile outside the $\mathrm{q}=1$ surface. Figure 18 (b) shows that the FEB result from the localized transport model agrees well also with the experimental energy spectrum of the central $I_{H X R}$.

An example of the electron distribution function in velocity space is presented in Figure 19 (a). Since ECCD is added in the direction of enhancing plasma current (co-ECCD), where $I_{p}<0$, the electron distribution function is significantly distorted in the direction of positive momentum $\left(p_{\|}>0\right)$. The radial distributions of suprathermal electrons are presented in Figure 19 (b,c), at different positions in the velocity phase space. As observed in the hard X-ray emission, the suprathermal electrons have a peak due to the localized ECCD, and this peak is more distinguished in the higher energy electrons, due to their lower collisionality. The spatial 

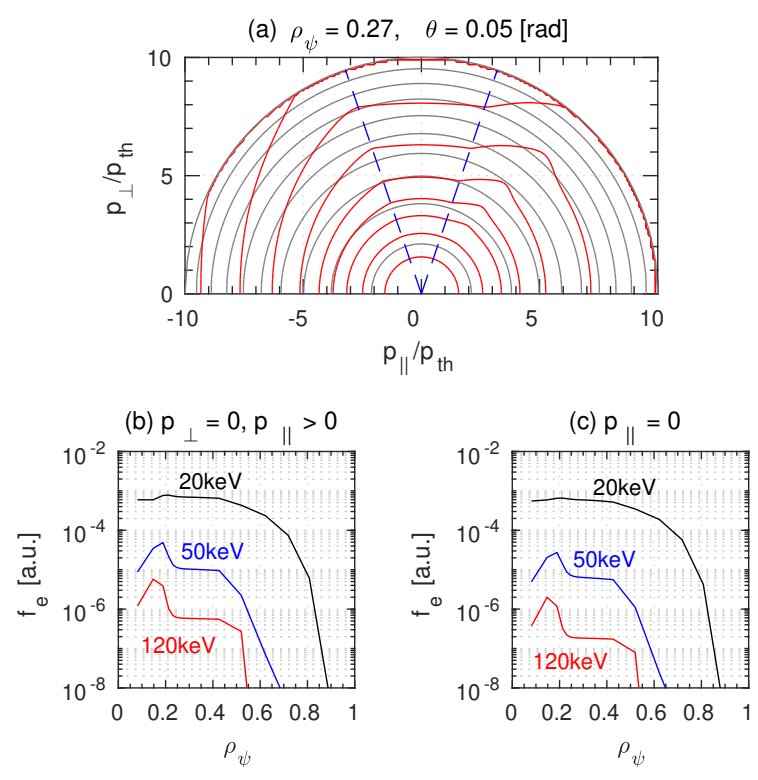

Figure 19: (a) 2-D contour plot of the electron distribution at $\rho_{\psi}=0.27$ and $\theta=0.05$. The concentric circles in grey correspond to the Maxwellian distribution. Radial profile of the electron distribution for different energies, selected along (b) $p_{\perp}=0, p_{\|}>0$ and (c) $p_{\|}=0$, of the 2-D distribution.
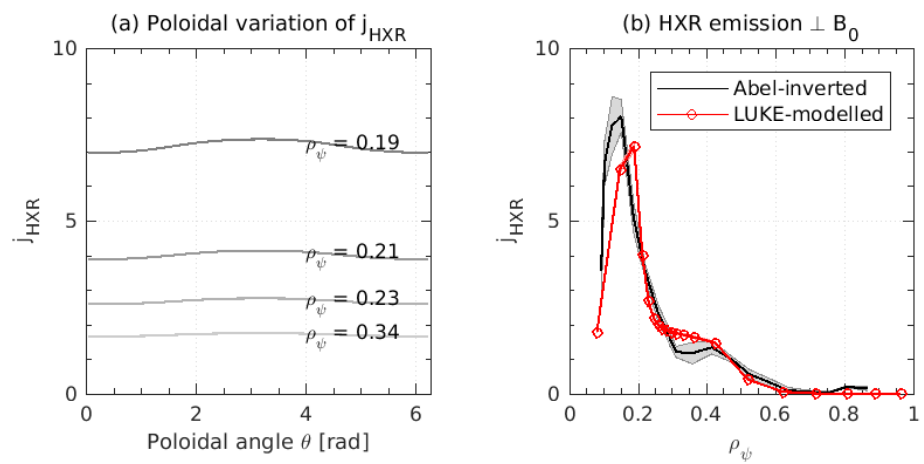

Figure 20: (a) The hard X-ray emission perpendicular to the magnetic field, (b) the modelled hard X-ray emission and Abel-inversion result comparison

gradient of the electron distribution function is negative $\left(\partial f_{e} / \partial \rho<0\right)$ except inside the ECCD peak, which is localized near the magnetic axis.

This electron distribution function is used to compute the Bremsstrahlung radiation in the hard X-ray synthetic diagnostic module [35]. The Bremsstrahlung emission in the perpendicular direction to the magnetic field, which is emitted in the poloidal plane, is presented in Figure 20 (a) as a function of the poloidal angle. The perpendicular hard X-ray emission is stronger at the high-field-side $(\theta=\pi)$ because of the magnetic moment as discussed in section 3.3, but the poloidal variation in $\theta$ is smaller than the variation in between different radial points in $\rho_{\psi}$. Thus it can be reasonable to use the Abel-inversion method in section 3.3, assuming a uniform emissivity at each flux surface. In Figure 20 (b), the modelled hard X-ray emission profile $j_{H X R}$ 
agrees well with the Abel-inverted profile from Figure 10.

\subsection{Solving the linear fishbone dispersion relation}

The Fokker-Planck modelled electron distribution function is used to solve the linear fishbone dispersion relation, $\delta \hat{W}_{f}+\delta \hat{W}_{k}(\omega)=\delta I(\omega)$, where $\omega$ is the complex frequency of the mode, $\delta \hat{W}_{f}$ and $\delta \hat{W}_{k}$ describe the fluid and kinetic components of the potential energy, respectively, and $\delta I$ includes the physics inside the inertial layer. The code MIKE is used [45], which was developed to solve the linear fishbone dispersion relation. This code is coupled to the Fokker-Planck code LUKE, such that the equilibrium profile and the numerical results from LUKE can be used in solving the linear dispersion relation. In practice, a free parameter $\delta \hat{W}_{c}$ is added to the dispersion relation to modify the fluid term; this parameter acts as a proxy for the ideal MHD $\delta \hat{W} . \delta \hat{W}_{f}$ and $\delta \hat{W}_{k}$ are decomposed into thermal and suprathermal particle parts.
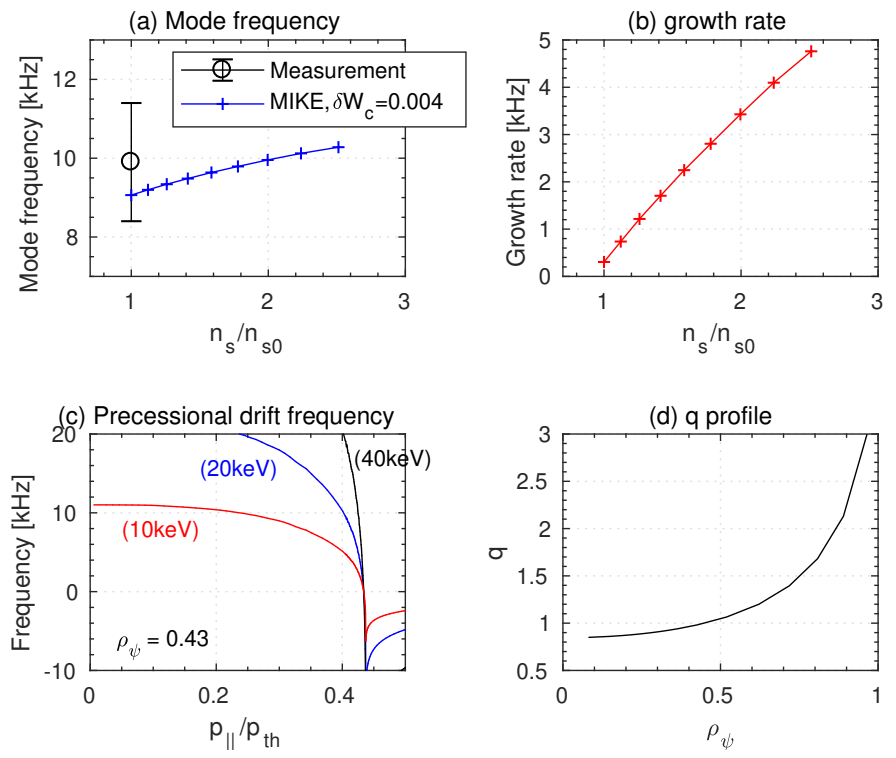

Figure 21: (a) mode frequency and (b) growth rate, are obtained for fixed $\delta \hat{W}_{c}=0.004$ and varying suprathermal electron population $n_{s}$. (c) Toroidal precessional drift frequency of suprathermal electrons near the $\mathrm{q}=1$ surface, as a function of pitch angle, $p_{\|} / p_{t h}$. (d) q profile of \#62609.

For the fixed $\delta \hat{W}_{c}$ value of 0.004 , the result of the calculation is presented in Figure $21(\mathrm{a}, \mathrm{b})$. In order to study the effect of the suprathermal electron population on the stability of the mode, the suprathermal electron density $n_{s}$ is scanned from $n_{s 0}$, which corresponds to the FokkerPlanck modelled electron distribution based on the experiment. In $\delta \hat{W}_{k}$, these suprathermal electrons are considered collisionless. The predicted mode frequency lies within the error range of the measured mode frequency, and the mode growth rate is positive and increases with $n_{s}$. Thus it is clear that the suprathermal electron population destabilizes the fishbone mode. Here the experimentally measured mode frequency is shifted related to the toroidal plasma rotation. Thus the measured mode frequency in the plasma frame can be compared to the frequency predicted by the simulation.

Previously the excitation of the internal kink was attributed to the resonant interaction of barely trapped/passing electrons $[1,7,11,14]$ or deeply trapped electrons $[9,10,48]$ with the mode. 
In the TCV experiment, the mode rotation directions obtained in the experiment and obtained in the simulation are in the electron diamagnetic drift direction. The calculation of the toroidal precessional drift frequency of suprathermal electrons near the $q=1$ surface, in Figure 21 (c), shows that the deeply and moderately trapped electrons rotate in the same direction as the mode. In addition, the q profile (21 (d)) is not flat in the $\mathrm{q}<1$ region. It is noted in [39] that although passing and trapped drift precession can be similar in order of magnitude, the passing electron contribution to the dispersion relation is significant only if $\Delta q \omega_{b} \sim\left\langle\omega_{d \psi}\right\rangle$, where $\omega_{b}$ and $\omega_{d \psi}$ are the bounce frequency and toroidal drift frequency, respectively. This for electrons is true only very close to $\mathrm{q}=1$, while the mode structure is not zero for $0<r<r_{q=1}$. For that reason we can neglect the role of passing electron (see page 9 of [39] for more details).

In Figure 22, the ion diamagnetic drift frequency $\omega_{* i}$ in the inertia term of the fishbone dispersion relation is scanned, with different $\delta \hat{W}_{c}$ values. The suprathermal electron density is set to $n_{s 0}$, the value based on the experiment. This shows that the electron fishbone mode that rotates opposite to the ion diamagnetic drift direction is stabilized by enhancing the ratio $\omega_{* i} / \omega_{* e}$, where $\omega_{* e}$ is the electron diamagnetic drift frequency. In the TCV experiment, the ratio $\omega_{* i} / \omega_{* e}$ is small $(\sim 1 / 4)$, and as a result the electron-diamagnetic-drift-directed fishbone is not stabilized by ion finite Larmor radius (FLR) effects. In contrast, the DIII-D electron fishbone reported earlier [1], where ECCD and NBI were used, could be stable to these electron-directed fishbones. The only channel available is the ion-diamagnetic-drift-directed fishbone mode that resonates with barely trapped electrons. In the DIII-D case an inverted spatial gradient of suprathermal electrons $\left(\partial f_{e} / \partial \rho>0\right)$ at $\mathrm{q}=1$ assists the mode drive.

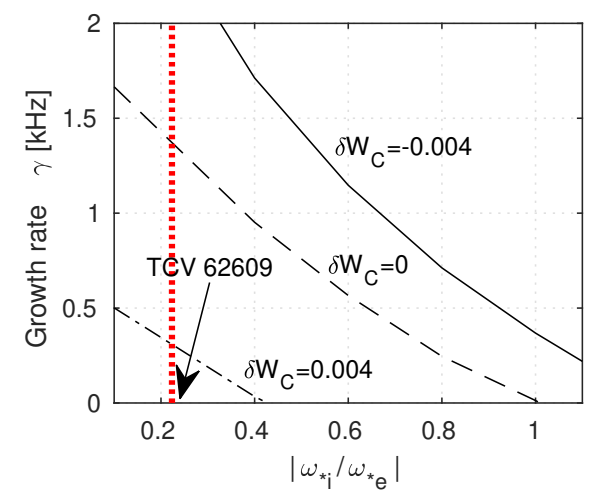

Figure 22: Growth rate obtained for varying $\omega_{* i}$ and $\delta \hat{W}_{c}$

In order to study the ECCD power effect on the onset of the fishbone mode, three time windows during the ECCD power scan experiment (\#62755, Figure 13), are selected for the analysis. Since the equilibrium and the electron density are kept constant throughout the discharge, the electron temperature and RF power are varied according to the experimentally measured values. The Fokker-Planck modeling is based on the analysis in section 5.1, using the localized transport model. The modelled electron distribution function at different energies is presented in Figure 23 , for the three time windows. As the ECCD power decreases with time, as observed in the hard X-ray profiles, the suprathermal electron population decreases in every energy bin.

The solution of the linear fishbone dispersion relation is compared with the experimentally observed mode frequencies during the ECCD power scan, in Figure 24 (a). The calculated mode frequencies are Doppler-shifted considering the toroidal mode rotation frequency, $4.1 \mathrm{kHz}$ in the plasma current direction, and they agree well with the experiment. In all time windows, the 

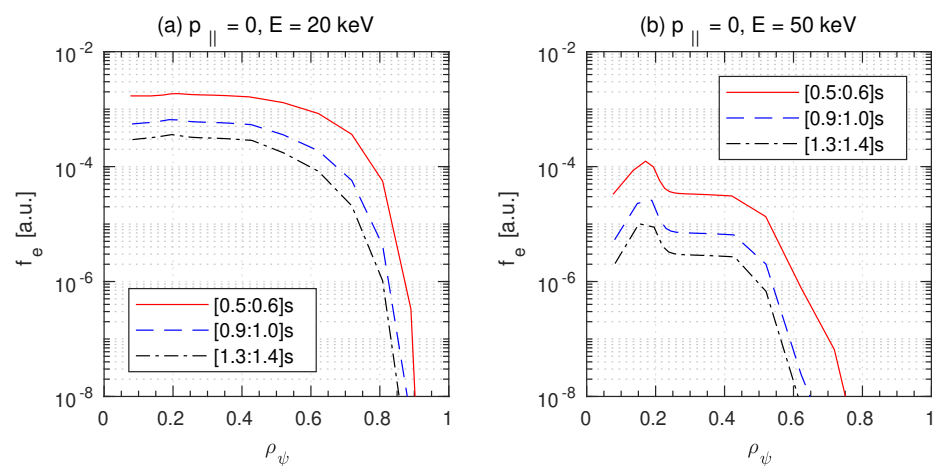

Figure 23: Radial profile of Fokker-Planck modelled electron distribution function, for $\theta=0$ and $p_{\|}=0$, at three time windows, from $0.5 \mathrm{~s}$ to $0.6 \mathrm{~s}$ when $P_{E C}=750 \mathrm{~kW}$, from $0.9 \mathrm{~s}$ to $1.0 \mathrm{~s}$ when $P_{E C}=550 \mathrm{~kW}$, from $1.3 \mathrm{~s}$ to $1.4 \mathrm{~s}$ when $P_{E C}=350 \mathrm{~kW}$.

growth rate is positive and the mode frequency increases when the suprathermal electron population $n_{s}$ increases. Thus it is clear that in this experiment the suprathermal electron population plays a destabilizing role and the mode frequency decreases as the population decreases owing to the ECCD power ramp-down.
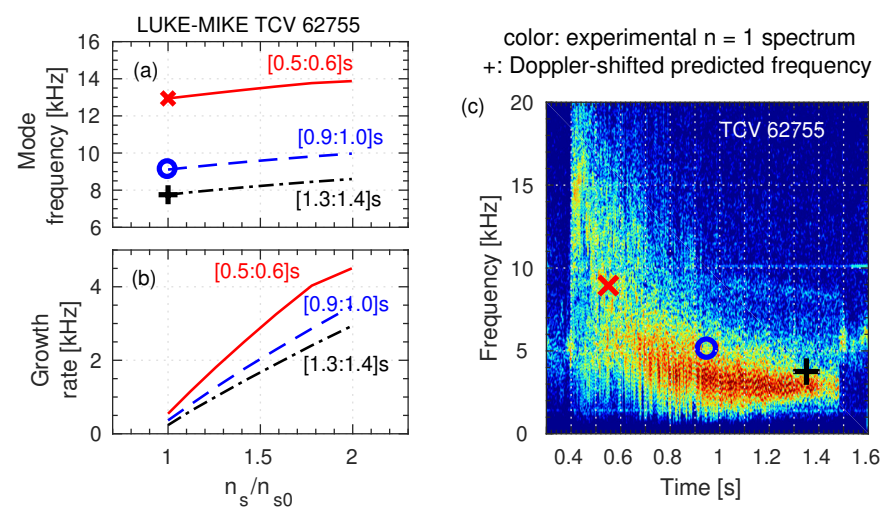

Figure 24: $(\mathrm{a}, \mathrm{b})$ The predicted mode frequency and growth rate in three time windows. (c) The Doppler-shifted mode frequency prediction is plotted on the $\mathrm{n}=1$ mode spectrogram from the experiment.

\section{Conclusions}

During ECCD injection in the TCV tokamak, fishbone-like bursts accompanied by central plasma relaxation are observed. The $\mathrm{m} / \mathrm{n}=1 / 1$ mode structure in the soft X-ray and hard X-ray profiles have been shown for the first time with a high temporal resolution comparable with the observed mode frequency. The soft X-ray and hard X-ray profiles are Abel-inverted, based on an equilibrium that includes a mode structure. The rotatable hard X-ray camera has enabled a study of different responses of trapped and passing electrons to the mode, separately.

The results have allowed us to see the evolution of the electron distribution related to the fishbone oscillations (Section 3). The mode is destabilized after the ramp-up of suprathermal electron (Figure 4). Then trapped suprathermal electrons preferentially interact with the mode and are expelled during the mode oscillation phase (Figure 11). The solution of the linear 
fishbone dispersion relation, based on the Fokker-Planck modeling of the electron distribution function coupled with the hard X-ray synthetic diagnostic, confirms the destabilization of the fishbone mode by deeply and moderately (not barely) trapped suprathermal electrons' resonant interaction with the mode (Section 5). This explains the observed mode rotation in the electron diamagnetic drift direction. The ECCD power scanning experiment and modeling have shown the mode frequency dependency on the electron distribution function, which can support the idea that the frequency chirping of a single fishbone burst is induced by the redistribution of the resonant electrons during the oscillation (Section 4).

Many questions regarding the stability of the trapped electron fishbone and its impact on the plasma remain. A systematic study of the non-linear growth phase is necessary to assess and predict the effect of the instability on the electron distribution, which may degrade the ECCD efficiency and impose constraints on ECCD scenarios. In the current experiment, the trapped electron fishbone has been obtained with monotonic pressure profile and relatively low ion temperature without NBI. However, this instability may be stabilized by the NBI-heated ion population, as suggested by Figure 22, in which case it may not be a serious obstacle in future tokamak operation with high ion temperature, such as ITER's burning plasma experiments. Future experiments in TCV will add NBI to the ECCD plasma with electron fishbones, in order to investigate the effect of ions on the instability. It might be expected that with the increase in ion temperature, the electron fishbone will no longer propagate in the electron diamagnetic direction, and the other branch of electron fishbones [1] may be generated due to resonance with barely trapped electrons.

\section{Acknowledgments}

This work was supported in part by the Swiss National Science Foundation. This work has been carried out within the framework of the EUROfusion Consortium and has received funding from the Euratom research and training programme 2014 - 2018 and 2019 - 2020 under grant agreement No 633053. The views and opinions expressed herein do not necessarily reflect those of the European Commission.

\section{References}

[1] KL Wong et al. Physical Review Letters, 85(5):996, 2000.

[2] M Valovic et al. Nuclear Fusion, 40(9):1569, 2000.

[3] JA Snipes et al. Nuclear Fusion, 48(7):072001, 2008.

[4] CB Deng et al. Physical Review Letters, 103(2):025003, 2009.

[5] M Isobe et al. Nuclear Fusion, 50(8):084007, 2010.

[6] XT Ding et al. Nuclear Fusion, 53(4):043015, 2013.

[7] X T Ding et al. Nuclear Fusion, 42(5):491, 2002.

[8] W Chen et al. Nuclear Fusion, 49(7):075022, 2009.

[9] W Chen et al. Nuclear Fusion, 50(8):084008, 2010.

[10] LM Yu et al. Nuclear Fusion, 57(3):036023, 2017.

[11] F Zonca et al. Nuclear Fusion, 47(11):1588, 2007. 
[12] R Cesario et al. Nuclear Fusion, 49(7):075034, 2009.

[13] A Macor et al. Physical Review Letters, 102(15):155005, 2009.

[14] ZO Guimarães-Filho et al. Nuclear Fusion, 52(9):094009, 2012.

[15] L Delgado-Aparicio et al. Physics of Plasmas, 22(5):050701, 2015.

[16] L Sugiyama et al. Physics of Plasmas, 25(8):082120, 2018.

[17] YP Zhang et al. Review of Scientific Instruments, 81(10):103501, 2010.

[18] S Coda et al. Nuclear Fusion, 57(10):102011, 2017.

[19] F Hofmann et al. Nuclear Fusion, 28(10):1871, 1988.

[20] J-M Moret et al. Review of Scientific Instruments, 69(6):2333-2348, 1998.

[21] M Anton et al. Plasma Physics and Controlled Fusion, 38(11):1849, 1996.

[22] T Dudok de Wit et al. Physics of Plasmas, 1(10):3288-3300, 1994.

[23] A Sushkov et al. Review of Scientific Instruments, 79(2):023506, 2008.

[24] VS Udintsev. In EC-14: 14th Joint Workshop on Electron Cyclotron Emission and Electron Cyclotron Resonance Heating, 2006.

[25] S Coda et al. In 1st EPS conference on Plasma Diagnostics, volume 240, page 139. SISSA Medialab, 2016.

[26] S Coda. Review of Scientific Instruments, 79(10):10F501, 2008.

[27] J Kamleitner et al. Nuclear Instruments and Methods in Physics Research Section A: Accelerators, Spectrometers, Detectors and Associated Equipment, 736:88-98, 2014.

[28] Y Peysson et al. Plasma Physics and Controlled Fusion, 54(4):045003, 2012.

[29] ZA Pietrzyk et al. Nuclear Fusion, 39(5):587, 1999.

[30] I Furno et al. Nuclear Fusion, 41(4):403, 2001.

[31] IT Chapman et al. Physical Review Letters, 105(25):255002, 2010.

[32] JP Graves et al. Plasma Physics and Controlled Fusion, 55(1):014005, 2012.

[33] IH Hutchinson. Cambridge University Press, 2005.

[34] S Texter et al. Nuclear Fusion, 26(10):1279, 1986.

[35] Y Peysson et al. Physics of Plasmas, 15(9):092509, 2008.

[36] R B White. World Scientific Publishing Company, 2013.

[37] RW Harvey et al. Physical review letters, 88(20):205001, 2002.

[38] S Coda et al. Nuclear fusion, 43(11):1361, 2003.

[39] J P Graves. Plasma Physics and Controlled Fusion, 55(7):074009, 2013.

[40] L Chen et al. Physical Review Letters, 52:1122-1125, 1984.

[41] CC Petty et al. Nuclear Fusion, 42(12):1366, 2002.

[42] T Suzuki et al. Nuclear Fusion, 44(7):699, 2004.

[43] J Decker. In AIP Conference proceedings, volume 694, pages 447-454. AIP, 2003.

[44] J Decker et al. Tech. rep. No EUR-CEA-FC-1736, 2004.

[45] A Merle et al. Physics of Plasmas, 19(7):072504, 2012.

[46] R W Harvey et al. Physical Review Letters, 88:205001, 2002.

[47] S Coda et al. Nuclear Fusion, 43(11):1361-1370, 2003.

[48] H He et al. Nuclear Fusion, 54(11):114001, 2014. 